\title{
Cognitive Rigidity, Habitual Tendencies, and Obsessive- \\ Compulsive Symptoms: Individual Differences and Compensatory Interactions
}

\author{
Smriti Ramakrishnan ${ }^{1}$, Trevor W Robbins ${ }^{2,3}$, \& Leor Zmigrod ${ }^{2,3, *}$ \\ ${ }^{1}$ School of Clinical Medicine, University of Cambridge \\ ${ }^{2}$ Department of Psychology, University of Cambridge \\ ${ }^{3}$ Behavioural and Clinical Neuroscience Institute, University of Cambridge \\ *Contact: Dr Leor Zmigrod, Department of Psychology, Cambridge, CB2 3EB, UK, \\ 1z343@cam.ac.uk.
}

This paper is currently under review. For any questions please contact Dr Leor Zmigrod, lz343@cam.ac.uk. The paper can be cited as:

Ramakrishnan, S., Robbins, T.W., \& Zmigrod, L. (under review). Cognitive Rigidity, Habitual Tendencies, and Obsessive-Compulsive Symptoms: Individual Differences and Compensatory Interactions. DOI: 10.31234/osf.io/82dbt

\begin{abstract}
Recent theories have posited a range of cognitive risk factors for obsessive-compulsive disorder (OCD), including cognitive inflexibility and a maladaptive reliance on habits. However, empirical and methodological inconsistencies have obscured the understanding of whether inflexibility and habitual tendencies indeed shape OCD symptoms in clinical and subclinical populations, and whether there are notable interactions amongst these traits. The present investigation adopted an individual differences approach to examine the associations between behaviourally-assessed cognitive flexibility and subclinical OCD symptomatology. It also explored the nature of the interactions between cognitive flexibility and habitual
\end{abstract}


tendencies, and the degree to which these cognitive traits predict subclinical OCD symptomatology. Across two studies, including a preregistration, Bayesian and regression analyses revealed that cognitive inflexibility and compulsive habitual tendencies act as unique and independent predictors of subclinical OCD symptomatology. Furthermore, there was a significant interaction between cognitive rigidity and habitual compulsivity, which accounted for $49.4 \%$ of the variance in subclinical OCD symptomatology in Study 1, and 37.3\% in Study

2. In-depth analyses revealed a compensatory effect between cognitive inflexibility and habitual compulsivity such that both are necessary for OCD symptomatology, but neither is sufficient. These results imply that in order to generate reliable and nuanced models of the endophenotype of OCD symptomatology, it is essential to account for interactions between psychological traits. Moreover, the present findings have important implications for theories on the cognitive roots of $\mathrm{OCD}$, and potentially in the development of interventions that target both cognitive inflexibility and habitual compulsivity.

\section{Keywords}

Cognitive rigidity; cognitive flexibility; habits; obsessive-compulsive; interactions; individual differences; Bayes Factors; subclinical symptoms. 


\section{Introduction}

Obsessive-compulsive disorder (OCD) is a highly debilitating condition affecting between 1.1 and $1.8 \%$ of the population worldwide (OCD UK, 2021). It is characterised by unpleasant, unwanted obsessive thoughts, and repetitive compulsive rituals to try and neutralise these thoughts, and as such, can severely impair all aspects of quality of life (Eisen et al., 2006). Elucidating the cognitive underpinnings of OCD is of great importance in improving our understanding of this condition to better help patients. In order to do this, we must appreciate the spectral nature of psychiatric conditions and the variability in their manifestations between individuals, and thus taking a dimensional approach is valuable. A clear evidence-based endophenotype may allow identification of individuals with a predisposition for OCD, and the development of more targeted screening measures and interventions. A number of theories have been proposed to explain the cognitive processes underlying OCD symptoms, and there have been several attempts to develop a unified account of the origins of OCD (e.g. Abramovitch \& Cooperman, 2015; Robbins et al., 2019), but as yet there are still substantial ambiguities and empirical inconsistencies.

Over the years, patterns of executive dysfunction in OCD have been extensively studied. These have suggested that deficits in certain domains of executive function, such as set shifting and inhibition, are relatively specific to OCD, while other domains such as planning, verbal fluency and working memory, are less impaired (Bannon et al., 2006; Abramovitch et al., 2021). Furthermore, these deficits in set shifting and inhibition are traitlike, "stable characteristics that are consistently deviant from normal even during remission" (Bannon et al., 2006, p.1032), meaning that they are independent of symptomatology, and thus may play an etiological role in the development or maintenance of OCD (Bannon et al., 2006). These findings are supported by neuroimaging studies demonstrating under-activation in dorsal 
frontal-striatal regions in OCD patients during task-switching, while healthy controls showed significant activation in these areas (Gu et al., 2008).

These findings have led to an interest in the study of excessive cognitive rigidity in OCD (e.g. Chamberlain et al., 2007; Meiran et al., 2011). Experimentally, cognitive flexibility has widely been shown to be impaired in OCD (e.g. Meiran et al., 2011), as well as in obsessive-compulsive personality disorder (OCPD) (Fineberg et al., 2015; Paast et al., 2016), the latter being characterized by extreme perfectionism, order and neatness, often associated with anxiety, with a prevalence of 3-8\% in adult populations (Diedrich \& Voderholzer 2015). However, impaired cognitive flexibility has not been demonstrated in other obsessivecompulsive spectrum disorders (OCSDs) such as trichotillomania (Chamberlain et al., 2006), or other psychiatric disorders such as generalised anxiety disorder (Rosa-Alcázar et al., 2020). This suggests that low cognitive flexibility may act as a specific predictor of OCD, providing support for cognitive flexibility accounts of OCD. Impaired cognitive flexibility has also been demonstrated in unaffected first-degree relatives of both adult (Chamberlain et al., 2007) and paediatric (Abramovitch et al., 2021) OCD patients, suggesting that it may be an endophenotype (a heritable, intermediate marker of risk between phenotype and genetics) of OCD. Furthermore, this may be specific to OCD, as cognitive flexibility does not seem to be an endophenotype for other psychiatric disorders such as schizophrenia (Ceaser et al., 2008). The combination of clinically observed rigidity, and trait-like and even endophenotypic cognitive inflexibility in OCD patients, accounts for the focus on cognitive inflexibility as a potential causal factor, and highlights the importance of further study in this area.

Nonetheless, there has been some dispute over the association between cognitive inflexibility and OCD, with some studies finding broad impairments in executive function in patients with OCD (Snyder et al., 2015; Shin et al., 2014), rather than specific deficits in cognitive flexibility. A meta-analysis conducted by Fradkin and colleagues (2018) found no 
evidence for impaired cognitive flexibility in OCD. However, this only included studies using measures of flexibility that are externally-cued (or reactive), such as set-shifting and taskswitching, in which the participant must switch by reacting to cues, but not measures of selfdirected (or generative) flexibility, in which the participant must internally generate the switching. Dissociations between performance on externally-cued and self-directed measures of cognitive flexibility have previously been demonstrated in patients with Parkinson's disease (Tomer et al., 2002), and furthermore, the two types of cognitive flexibility have distinct neural correlates (Eslinger and Grattan, 1993). Therefore, it may be that different paradigms in fact measure different aspects of the cognitive flexibility construct, and that only certain aspects of cognitive flexibility are impaired in OCD.

Another possible explanation for these conflicting findings may be that patients with clinical OCD often show more general cognitive impairment due to comorbidity with other forms of psychiatric disorder (Caudek et al., 2020). It is thus of great value to study cognitive flexibility in subclinical populations to eliminate this confounding factor. In addition, as clinical and subclinical obsessive-compulsive symptoms share similar features and are continuous with one another (Caudek et al., 2020), cognitive inflexibility in subclinical OCD may be a predisposing factor for the development of OCD. Impaired cognitive flexibility has been demonstrated in subclinical OCD by Sternheim and colleagues (2014), using a set-shifting task and a self-report measure of cognitive flexibility. Although this study provides strong support for the cognitive inflexibility specificity hypothesis of OCD, the participants were all female students within a narrow age range. Student populations can be expected to show relatively high levels of cognitive ability, and so the sample may not be representative of the general population. Additionally, this study used an externally-cued, rather than self-directed, paradigm to measure behavioural flexibility. The present study aims to demonstrate the relationship between cognitive flexibility and subclinical OCD symptoms in more 
representative samples, using a self-directed, generative measure of cognitive flexibility, the Alternative Uses Task, AUT (Guildford, 1967; Ionescu, 2012; Zmigrod et al., 2019; Zmigrod, 2020). As well as flexibility, the AUT is also scored on elaboration, fluency and originality, which can act as controls for other cognitive processes, ensuring that inferences are based on flexibility specifically and not general cognitive ability or fluency.

Another longstanding theory of the cognitive origins of OCD, proposed by Graybiel and Rauch (2000), is that OCD may be a clinical manifestation of an over-reliance on habits, involving maladaptive habit learning. Studies in both animals and humans have found evidence for a shift from goal-directed to habitual control in OCD, using behavioural measures such as outcome devaluation (Gillan et al., 2011) and contingency degradation (Vaghi et al., 2019), as well as self-report measures of habits (Ferreira et al., 2017). However, very few studies have explored how individual differences in habitual tendencies may be associated with subclinical OCD traits in the general population (see Ramakrishnan, Robbins, \& Zmigrod, 2021), and this analysis is crucial in order to augment our understanding of preliminary or predisposing OCD traits.

An apparent interplay between the different neural and cognitive mechanisms underlying OCD has led to suggestions that an interaction of several factors underlies OCD and its subtypes (Robbins et al., 2019). This dimensional model suggests that the interaction of three factors (anxiety or emotional vulnerability, cognitive inflexibility, and an imbalance between goal-directed and habitual control over behaviour) results in the development of OCD (Robbins et al., 2019). To the best of our knowledge, previous studies have not explored whether this interaction is present, and if so, whether it may have a role in predicting or screening for clinical OCD. Therefore, the present study aims to determine whether individual differences in habitual tendencies and cognitive flexibility may interact to predict subclinical OCD symptomatology. 
Consequently, the objectives of the present study were twofold: firstly, to examine the extent to which behaviourally-assessed cognitive flexibility is negatively related to subclinical OCD symptomatology; and secondly, to explore the nature of any interactions between habitual tendencies and cognitive flexibility to study whether these, in conjunction, may predict OCD traits.

\section{Study 1}

Study 1 sought to investigate associations between cognitive flexibility, subclinical OCD symptomatology, and the HTQ, in order to improve our understanding of the cognitive processes underlying OCD traits in the general population.

We addressed two main hypotheses as follows:

H1 - Cognitive flexibility is negatively correlated with levels of OCD traits (as cognitive flexibility has been shown to be impaired in subclinical OCD, e.g. Sternheim et al., 2014) or OCPD (e.g. Fineberg et al., 2015).

$\boldsymbol{H 2}$ - Habitual tendencies measured by the HTQ interact with cognitive flexibility in predicting OCD traits (due to OCD involving an over-reliance on habits, e.g. Graybiel and Rauch., 2000).

\section{$\underline{\text { Methods }}$}

\section{Participants}

For Study 1, 165 participants were recruited through Amazon Mechanical Turk (MTurk) online platform, which is well established in obtaining samples of the general population (Cheung et al., 2017), and each participant was paid $\$ 4.50$. Prior to data analysis, 35 participants $(21.2 \%)$ were removed due to failure of attention checks and repeat participation in the study identified via repeated answers in the Alternative Uses Task (AUT) and by duplicated IP addresses. 130 participants remained, consisting of $49 \%$ males, 50\% 
females and $1 \%$ other, between the ages of 22 and $73(\mathrm{M}=39.527, \mathrm{SD}=12.120)$, all of whom were based in the United States of America. The sample population identified as $72 \%$ White, 11\% Mixed ethnicity, 8\% Black or African American, 6\% Asian, 2\% American Indian or Alaska Native, and 1\% Hispanic/Latino. The highest stages of educational attainment of the sample were as follows: $1 \%$ had achieved less than a high school degree, $13 \%$ had graduated high school, 19\% had completed some school but did not have a degree, $15 \%$ had completed a 2-year Associate degree in college, 38\% had completed a 4-year Bachelor's degree in college, $12 \%$ had a Master's degree, and 2\% had a Doctoral or Professional degree. Ethical approval for the study was acquired from the Department of Psychology Ethics Committee of the University of Cambridge. In line with the Declaration of Helsinki (1964), electronic informed consent was obtained from all participants before beginning the survey, and participants were notified that they may terminate their participation in the study at any point.

\section{Measures}

We administered the measures in the form of an electronic survey. In order to measure habitual tendencies, we used the 11-item HTQ, which was rated on 7-point Likert scales ranging from "Strongly disagree" to "Strongly agree", and had an acceptable Cronbach's $\alpha$ value of 0.764 . To measure subclinical OCD symptomatology, we used the 18 -item revised version of the Obsessive-Compulsive Inventory, OCI (Foa et al., 2002), which was rated on 5point Likert scales ranging from "Not at all" to "Extremely", and had a high Cronbach's $\alpha$ value of 0.954. Example items include: "I check things more often than necessary", and "I am upset by unpleasant thoughts that come into my mind against my will". In order to measure cognitive flexibility and divergent thinking, we used the Alternative Uses Task (AUT), in which participants have 90 seconds in which to name as many different uses of a particular object as they can. In our survey, we administered two rounds of this task, using a brick and a 
newspaper as the objects, and responses were scored on flexibility, elaboration, fluency and originality, with high average inter-rater reliabilities of $0.88,0.84,0.97$ and 0.93 respectively. The AUT had a high Cronbach's $\alpha$ value of 0.885 . The survey also included two interspersed measures of attention to ensure that participants were concentrating on their responses to the questions ("I am paying attention to this survey. I strongly agree").

\section{$\underline{\text { Results }}$}

All statistical analyses were conducted using JASP (Version 0.12.2; JASP Team, 2020), SPSS (Version 27.0; IBM Corp, 2020) and R Studio (RStudio Team, 2020).

\section{Correlational analysis}

In order to consider any confounding variables, we examined the correlations between the demographic variables and the psychological variables of interest. Age was found to be negatively correlated with the OCI $(r=-0.236, p=0.007)$, and positively correlated with AUT

elaboration $(r=0.291, p<0.001)$ and AUT originality $(r=0.191, p=0.030)$. Gender differences were present for Preference for Regularity, $t(127)=-2.892, p=0.005$, with females scoring more highly than males. Educational attainment was negatively correlated with Preference for Regularity $(r=-0.226, p=0.010)$. Therefore, the demographic variables of age, gender and educational attainment were included as covariates in further analyses.

\section{Relationship between cognitive flexibility and subclinical OCD traits}

In order to evaluate the relationships between cognitive flexibility and subclinical OCD symptomatology, we computed the Pearson's correlations for these variables (see Table 1). As evident in Table 1 and Figure 1, there was a significant negative correlation between AUT Flexibility and the OCI $(r=-0.390, p<0.001)$, as hypothesised, suggesting that individuals 
with increased subclinical OCD traits have a tendency towards increased cognitive rigidity, or decreased cognitive flexibility. The Pearson's $r$ effect size of -0.390 is relatively large, as per the individual differences research guidelines set out by Gignac and Szodorai (2016).

To complement the Pearson's correlations, we also examined the Bayes Factors (see Table 1), which quantify the evidential strength in favour of a significant correlation given the present data $\left(\mathrm{H}_{1}\right.$, the alternative hypothesis), or in favour of no significant correlation given the present data $\left(\mathrm{H}_{0}\right.$, the null hypothesis). In line with the guidelines by Wagenmakers and colleagues (2018), a Bayes Factor $\left(\mathrm{BF}_{10}\right)$ above 100 indicates "extreme evidence" for $\mathrm{H}_{1}$ (significant correlation). Here we found that the relationship between AUT Flexibility and the OCI possesses an extremely large Bayes Factor of 3557.577 (see Table 1), indicating that the observed data is 3557.577 times more likely under $\mathrm{H}_{1}$ than $\mathrm{H}_{0}$.

\section{Relationship between HTQ and subclinical OCD traits}

There was a significant positive correlation between habitual tendencies, measured by the HTQ, and subclinical OCD traits, measured by the OCI $(r=0.484, p<0.001)$. Of the three HTQ subscales, Compulsivity showed the strongest correlation with the OCI $(r=0.598, p<$ 0.001 ), and this relationship possessed an extremely large Bayes Factor of $1.474 \times 10^{11}$ (see Table 1), indicating that the observed data is $1.474 \times 10^{11}$ times more likely under $\mathrm{H}_{1}$ (significant correlation) than $\mathrm{H}_{0}$ (no correlation). As this Bayes Factor value is above 100, it indicates "extreme evidence" for $\mathrm{H}_{1}$, in line with the guidelines from Wagenmakers and colleagues (2018). 
Table 1. Correlation matrix of habitual tendencies, OCD traits and cognitive measures, including Pearson's correlations and Bayes Factors.

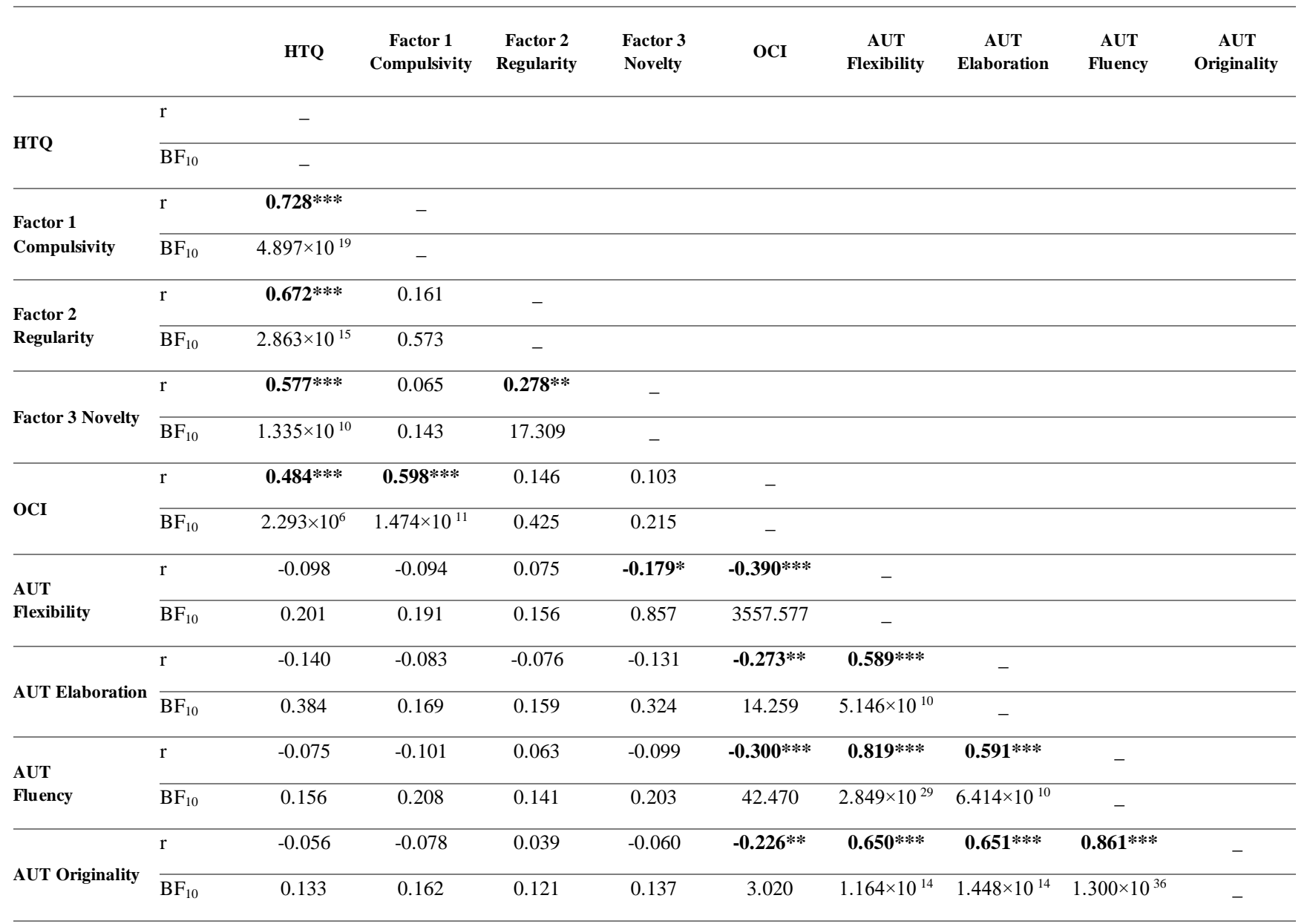

$* p<0.05, * * p<0.01, * * * p<0.001 ; \mathrm{BF}<3=$ Anecdotal evidence; $\mathrm{BF}<10=$ Moderate evidence; $\mathrm{BF}<30=\mathrm{Strong}$ evidence; $\mathrm{BF}<100=\mathrm{Very}$ strong evidence; $\mathrm{BF}>\mathbf{1 0 0}=$ Extremely strong evidence.

HTQ = Habitual Tendencies Questionnaire. OCI = Obsessive-Compulsive Inventory. AUT = Alternative Uses Task. 


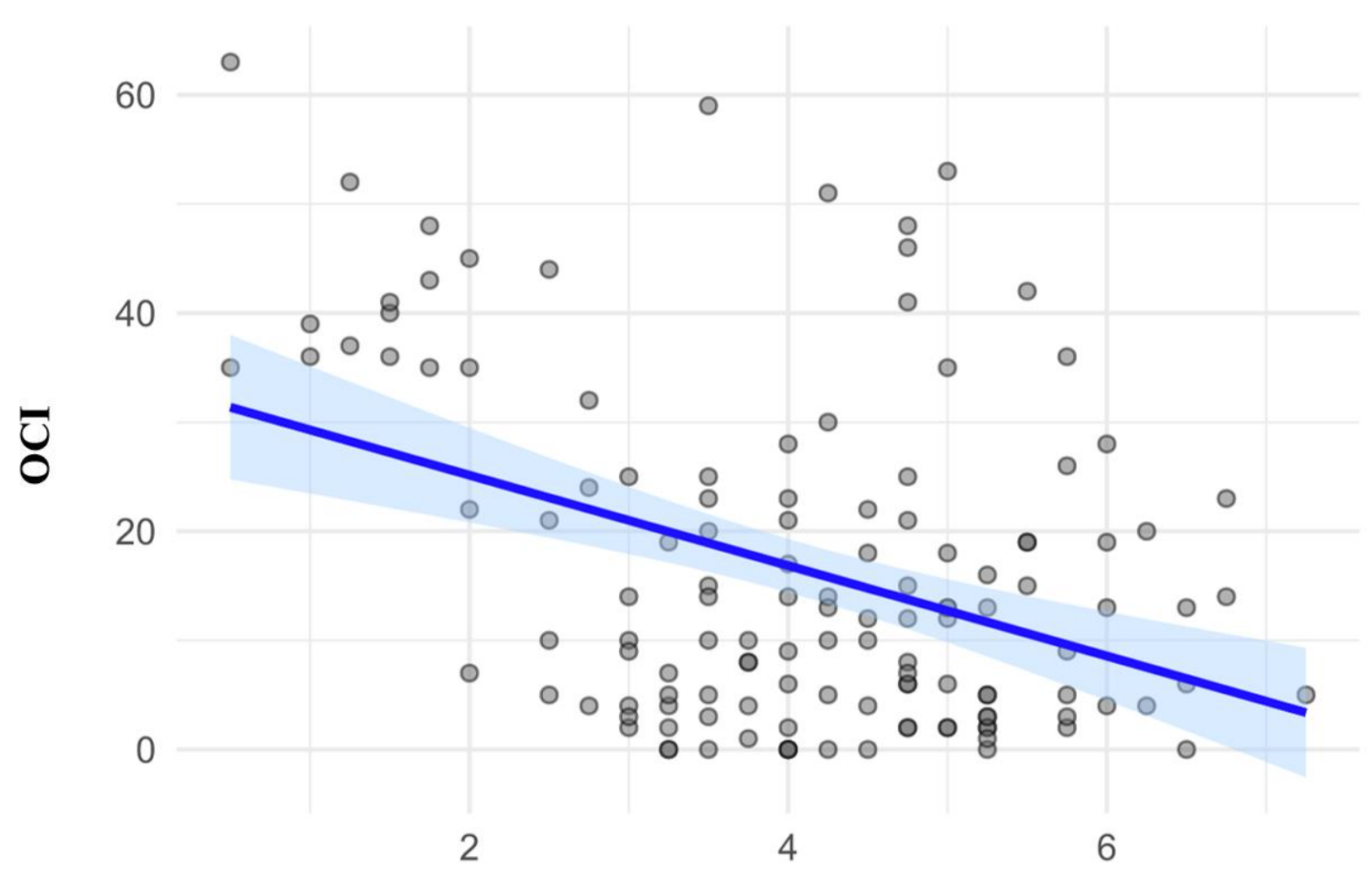

AUT Flexibility

Figure 1. Scatter plot showing correlations and between the Obsessive-Compulsive Inventory (OCI) and the Flexibility component of the Alternative Uses Task (AUT).

\section{Cognitive predictors of subclinical OCD symptomatology}

A simultaneous hierarchical regression was conducted with the three HTQ subscales and the four AUT components as predictors of subclinical OCD symptomatology (see Table 2). Both HTQ Compulsivity and AUT Flexibility emerged as significant predictors of the OCI. Higher Compulsivity and lower cognitive flexibility predicted greater levels of subclinical OCD traits. 
Table 2. Multiple regression with three Habitual Tendencies Questionnaire (HTQ) subscales and four Alternative Uses Task (AUT) components as predictors of subclinical OCD symptomatology (as measured by the Obsessive-Compulsive Inventory, OCI), with demographic variables age, gender and educational attainment as covariates.

\begin{tabular}{|c|c|c|c|c|c|c|c|c|}
\hline \multicolumn{2}{|r|}{ Dependent Variable: OCI } & \multicolumn{7}{|c|}{ Coefficients } \\
\hline \multirow{2}{*}{ Model } & & \multicolumn{2}{|c|}{ Unstandardised Coefficients } & \multirow{2}{*}{$\begin{array}{c}\begin{array}{c}\text { Standardised } \\
\text { Coefficients }\end{array} \\
\beta\end{array}$} & \multirow[b]{2}{*}{$t$} & \multirow[b]{2}{*}{$p$} & \multicolumn{2}{|c|}{$95 \%$ Confidence Interval for $B$} \\
\hline & & $B$ & Standard Error & & & & Lower Bound & Upper Bound \\
\hline \multirow{5}{*}{ Step 1} & (Constant) & 24.646 & 6.475 & & 3.807 & 0.000 & 11.831 & 37.461 \\
\hline & Age & -0.294 & 0.112 & -0.233 & -2.616 & $0.010^{*}$ & -0.516 & -0.071 \\
\hline & Gender & -0.204 & 2.729 & -0.007 & -0.075 & 0.940 & -5.605 & 5.197 \\
\hline & Educational Attainment & 1.165 & 0.997 & 0.102 & 1.169 & 0.245 & -0.808 & 3.137 \\
\hline & $R^{2}=0.065 ; \mathrm{F}(3,124)=2.853, p=0.040^{*}$ & & & & & & & \\
\hline \multirow{12}{*}{ Step 2} & (Constant) & 12.440 & 7.073 & & 1.759 & 0.081 & -1.568 & 26.447 \\
\hline & Age & -0.146 & 0.091 & -0.116 & -1.609 & 0.110 & -0.325 & 0.034 \\
\hline & Gender & -0.148 & 2.159 & -0.005 & -0.069 & 0.945 & -4.424 & 4.127 \\
\hline & Educational Attainment & 0.546 & 0.808 & 0.048 & 0.675 & 0.501 & -1.055 & 2.146 \\
\hline & HTQ Compulsivity & 1.425 & 0.188 & 0.523 & 7.596 & $<0.001 * * *$ & 1.053 & 1.796 \\
\hline & HTQ Regularity & 0.414 & 0.283 & 0.111 & 1.464 & 0.146 & -0.146 & 0.974 \\
\hline & HTQ Novelty & -0.041 & 0.293 & -0.010 & -0.140 & 0.889 & -0.622 & 0.540 \\
\hline & AUT Flexibility & -4.344 & 1.347 & -0.408 & -3.224 & $0.002 * *$ & -7.013 & -1.676 \\
\hline & AUT Elaboration & -0.192 & 0.746 & -0.025 & -0.258 & 0.797 & -1.670 & 1.285 \\
\hline & AUT Fluency & 0.417 & 0.958 & 0.078 & 0.435 & 0.664 & -1.480 & 2.313 \\
\hline & AUT Originality & 0.283 & 1.009 & 0.042 & 0.280 & 0.780 & -1.715 & 2.281 \\
\hline & $R^{2}=0.491 ; \mathrm{F}(7,117)=14.021, p<0.001 * * *$ & & & & & & & \\
\hline
\end{tabular}

As shown in Table 2, HTQ Compulsivity and cognitive flexibility were significant and unique predictors of the OCI. In order to examine whether there was a significant interaction between these two predictors, hierarchical linear regression was then conducted (see Table 3). In Step 1, the demographic variables age, gender and educational attainment were entered as covariates. As shown in Table 3, age was a significant negative predictor of subclinical OCD symptomatology, such that older participants exhibited lower levels of subclinical OCD symptomatology than younger participants in the present sample. In Step 2, HTQ compulsivity and cognitive flexibility were added, both of which were significant predictors of subclinical OCD symptomatology. HTQ Compulsivity had a positive relationship with the OCI $(\beta=0.542$, 
$p<0.001)$ and cognitive flexibility had a negative relationship with the OCI $(\beta=-0.324, p<$

0.001). These independent variables accounted for a significant proportion of the variance in subclinical OCD symptomatology $\left(r^{2}=0.476\right)$. In Step 3, the interaction term for HTQ Compulsivity and cognitive flexibility was entered. There was a significant interaction effect between compulsivity and cognitive flexibility, as shown in Table 3 , with $\beta=-0.547, p=0.039$.

The interaction term increased the $r^{2}$ value to 0.494 , thus accounting for a further $1.8 \%$ of the variance in subclinical OCD symptomatology.

Table 3. 3-step hierarchical linear regression with Habitual Tendencies Questionnaire (HTQ) Compulsivity, Alternative Uses Task (AUT) Flexibility and the interaction term between them as predictors of subclinical OCD symptomatology (as measured by the Obsessive-Compulsive Inventory, OCI), with demographic variables age, gender and educational attainment as covariates.

\begin{tabular}{|c|c|c|c|c|c|c|c|c|}
\hline \multicolumn{2}{|r|}{ Dependent Variable: OCI } & \multicolumn{7}{|c|}{ Coefficients } \\
\hline \multirow{2}{*}{ Model } & & \multicolumn{2}{|c|}{ Unstandardised Coefficients } & \multirow{2}{*}{$\begin{array}{c}\begin{array}{c}\text { Standardised } \\
\text { Coefficients }\end{array} \\
\beta\end{array}$} & \multirow[b]{2}{*}{$t$} & \multirow[b]{2}{*}{$p$} & \multicolumn{2}{|c|}{$95 \%$ Confidence Interval for $B$} \\
\hline & & $B$ & Standard Error & & & & Lower Bound & Upper Bound \\
\hline \multirow{5}{*}{ Step 1} & (Constant) & 24.646 & 6.475 & & 3.807 & 0.000 & 11.831 & 37.461 \\
\hline & Age & -0.294 & 0.112 & -0.233 & -2.616 & $0.010^{*}$ & -0.516 & -0.071 \\
\hline & Gender & -0.204 & 2.729 & -0.007 & -0.075 & 0.940 & -5.605 & 5.197 \\
\hline & Educational Attainment & 1.165 & 0.997 & 0.102 & 1.169 & 0.245 & -0.808 & 3.137 \\
\hline & $R^{2}=0.065 ; \mathrm{F}(3,124)=2.853, p=0.040^{*}$ & & & & & & & \\
\hline \multirow{7}{*}{ Step 2} & (Constant) & 16.485 & 5.944 & & 2.773 & 0.006 & 4.719 & 28.252 \\
\hline & Age & -0.131 & 0.086 & -0.104 & -1.518 & 0.132 & -0.302 & 0.040 \\
\hline & Gender & 0.711 & 2.063 & 0.023 & 0.345 & 0.731 & -3.372 & 4.795 \\
\hline & Educational Attainment & 0.414 & 0.757 & 0.036 & 0.547 & 0.586 & -1.084 & 1.912 \\
\hline & HTQ Compulsivity & 1.475 & 0.182 & 0.542 & 8.093 & $<0.001 * * *$ & 1.114 & 1.836 \\
\hline & AUT Flexibility & -3.453 & 0.711 & -0.324 & -4.854 & $<0.001 * * *$ & -4.861 & -2.045 \\
\hline & $R^{2}=0.476 ; \mathrm{F}(2,122)=47.904, p<0.001 * * *$ & & & & & & & \\
\hline \multirow{8}{*}{ Step 3} & (Constant) & 2.076 & 9.054 & & 0.229 & 0.819 & -15.848 & 20.000 \\
\hline & Age & -0.146 & 0.086 & -0.116 & -1.703 & 0.091 & -0.315 & 0.024 \\
\hline & Gender & 0.275 & 2.046 & 0.009 & 0.134 & 0.893 & -3.776 & 4.325 \\
\hline & Educational Attainment & 0.329 & 0.748 & 0.029 & 0.440 & 0.661 & -1.151 & 1.809 \\
\hline & HTQ Compulsivity & 2.665 & 0.597 & 0.978 & 4.461 & $<0.001 * * *$ & 1.483 & 3.848 \\
\hline & AUT Flexibility & 0.268 & 1.915 & 0.025 & 0.140 & 0.889 & -3.523 & 4.059 \\
\hline & HTQ Compulsivity x AUT Flexibility & -0.281 & 0.135 & -0.547 & -2.089 & $0.039 *$ & -0.547 & -0.015 \\
\hline & $R^{2}=0.494 ; \mathrm{F}(1,121)=4.363, p=0.039^{*}$ & & & & & & & \\
\hline
\end{tabular}




\section{Interaction effects between habitual compulsivity, cognitive flexibility and subclinical}

\section{OCD traits}

We then conducted simple slope analyses (SSA) to investigate the association between cognitive flexibility and subclinical OCD symptomatology at 1 standard deviation (SD) above and below mean HTQ Compulsivity, with age, gender and educational attainment as covariates (see Figure 2a). A significant negative relationship was found between cognitive flexibility and subclinical OCD symptomatology when HTQ Compulsivity was high (at $+1 \mathrm{SD}, b=-4.52, p$ $<0.001$ ), while no significant relationship was found when HTQ Compulsivity was low (at -1 $\mathrm{SD}, b=-1.36, p=0.27)$. Carrying out the reciprocal SSA to investigate the association between HTQ Compulsivity and subclinical OCD symptomatology (see Figure 2b) demonstrated that there were significant positive relationships between HTQ Compulsivity and subclinical OCD symptomatology both when cognitive flexibility was high (at $+1 \mathrm{SD}, b=1.12, p<0.001$ ), and when it was low (at $-1 \mathrm{SD}, b=1.92, p<0.001$ ). The interaction effects between HTQ Compulsivity and cognitive flexibility (measured by the AUT) are shown in the filled contour plot in Figure 2c. This shows that the relationship between HTQ Compulsivity and subclinical OCD symptomatology varies depending on cognitive flexibility, such that at high levels but not low levels of HTQ Compulsivity, cognitive flexibility differentiates between high and low levels of subclinical OCD symptomatology. It also shows that the relationship between cognitive flexibility and subclinical OCD symptomatology varies depending on HTQ Compulsivity, such that at both high and low levels of cognitive flexibility, HTQ Compulsivity differentiates between high and low levels of subclinical OCD symptomatology. The highest levels of subclinical OCD traits were observed in participants with high HTQ Compulsivity scores and low AUT Flexibility scores, indicating a compensatory effect, in accordance with the significant interaction effect shown in the hierarchical linear regression (see Table 3). Meanwhile, the lowest levels of subclinical OCD traits were observed in participants with low 
HTQ Compulsivity scores, regardless of their AUT Flexibility scores. Therefore, high HTQ

Compulsivity and low cognitive flexibility are necessary for the highest levels of subclinical OCD symptomatology, while neither is sufficient independently. These findings are in line with those from the SSA analyses. We used the Johnson-Neyman technique to analyse this interaction further (Johnson and Neyman, 1936). This technique complements simple slope analysis, as it calculates the specific values of the moderator at which the predictor transitions from non-significant to significant, rather than using potentially arbitrary conditional values of the moderator (Preacher et al., 2006). This indicated that the association between cognitive flexibility and OCD was significantly negative at compulsivity scores of 8.35 and above (see Figure 2d).

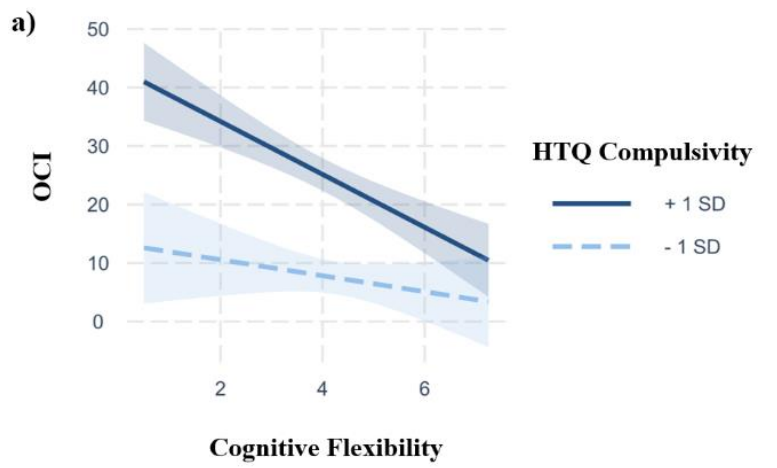

c)

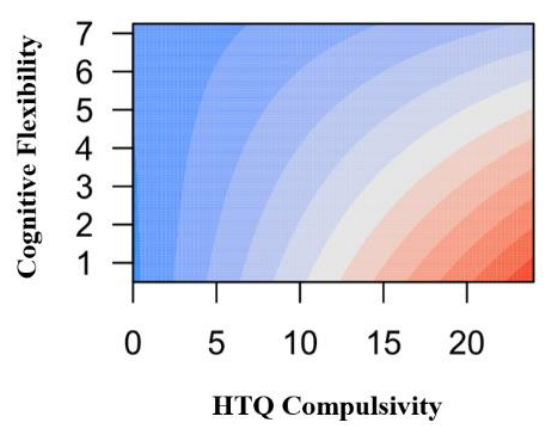

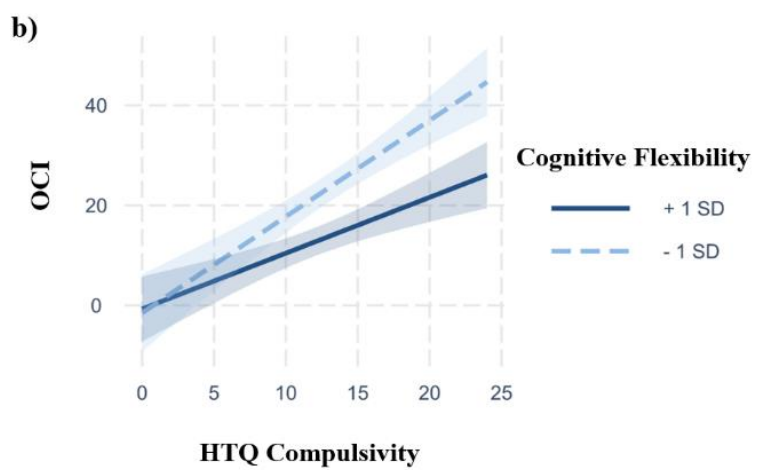

d)

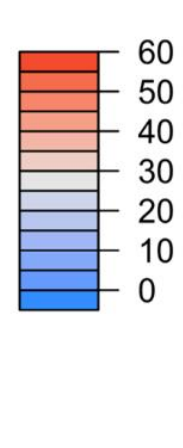

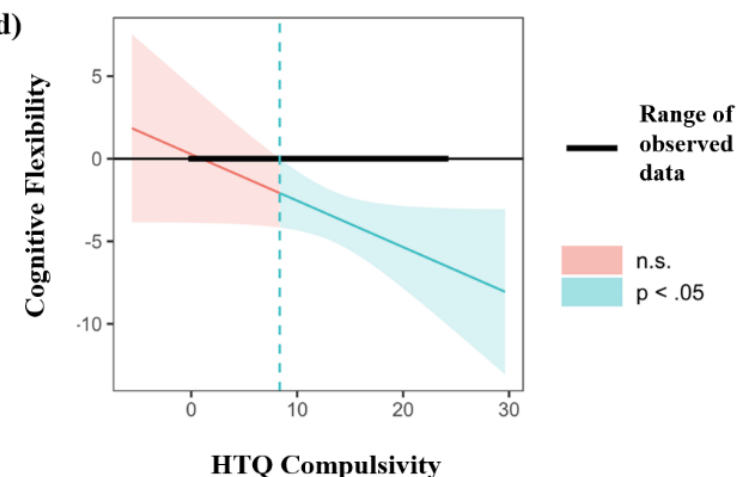

Figure 2. a) Interaction plot between the Compulsivity subscale of the Habitual Tendencies Questionnaire (HTQ), cognitive flexibility (as measured by the Alternative Uses Task) and subclinical OCD symptomatology (as measured by the Obsessive-Compulsive Inventory, OCI) at $1 \mathrm{SD}$ above and below the mean, controlling for age, gender and educational attainment, with cognitive flexibility as the predictor and HTQ Compulsivity as the moderator. Created using the interactions and interplot packages in the statistical software R Studio. b) Interaction 
plot between the Compulsivity subscale of the Habitual Tendencies Questionnaire (HTQ), cognitive flexibility (as measured by the Alternative Uses Task) and subclinical OCD symptomatology (as measured by the Obsessive-Compulsive Inventory, OCI) at $1 \mathrm{SD}$ above and below the mean, controlling for age, gender and educational attainment, with HTQ Compulsivity as the predictor and cognitive flexibility as the moderator. (Created using the interactions and interplot packages in the statistical software R Studio.) c) Representation of the regression surface predicting subclinical OCD symptomatology (as measured by the Obsessive-Compulsive Inventory, OCI) as a function of the Compulsivity subscale of the Habitual Tendencies Questionnaire (HTQ) and cognitive flexibility (as measured by the Alternative Uses Task), while controlling for age, gender and educational attainment. (Created using the visreg package in the statistical software R Studio.) d) Johnson-Neyman plot showing the conditional relation between cognitive flexibility and OCD symptomatology as a function of the Compulsivity subscale of the Habitual Tendencies Questionnaire (HTQ). The solid diagonal line represents the regression coefficient of cognitive flexibility (as measured by the Alternative Uses Task) for OCD symptomatology along the compulsivity spectrum. The dashed vertical line at a HTQ Compulsivity value of 8.35 represents the transition from significance to non-significance. The width of the regions reflects the $95 \%$ confidence intervals. (Created using the interactions and interplot packages in the statistical software $\mathrm{R}$ Studio.)

\section{Interim Discussion}

Study 1 has demonstrated that both cognitive inflexibility and compulsive habitual tendencies act as significant independent predictors of subclinical OCD symptomatology. Furthermore, hierarchical regression showed that cognitive inflexibility and compulsive habitual tendencies interact, accounting for a significant proportion of the variance in OCD traits. Simple slope analyses further defined this interaction, revealing that the negative relationship between cognitive flexibility and OCD traits was significant at high but not low levels of habitual compulsivity, suggesting that habitual compulsivity acts as a moderator of the association between cognitive inflexibility and subclinical OCD symptomatology. Finally, as shown in Figure 2c, the highest levels of OCD traits were observed in individuals with low cognitive flexibility and high habitual compulsivity, implying a compensatory effect. Therefore, both low levels of cognitive flexibility and high levels of compulsive habitual tendencies are necessary for the highest levels of subclinical OCD symptoms. 


\section{Study 2}

Study 1 demonstrated that cognitive inflexibility and habitual compulsivity independently predict subclinical symptomatology, and that habitual compulsivity moderates the negative association between cognitive flexibility and OCD traits, such that it is significant at high but not low levels of habitual compulsivity. Study 2 was conducted in order to reproduce the findings of Study 1 in a larger sample. The aims of Study 2 were as follows: (1) to replicate the relationships of cognitive flexibility and compulsive habitual tendencies with OCD traits (as measured by the OCI); (2) to replicate the interaction effect between cognitive flexibility and compulsive habitual tendencies in predicting OCD traits.

Study 2 was preregistered on the Open Science Framework at the following link as "Hypothesis \#5": https://osf.io/3ag79/?view_only=b9fd67d034c7425dbf559c05d2421cb3 . After the preregistration, some changes were made as follows: the data for binge eating, alcohol addiction, smoking habits and apathy were not analysed in relation to habitual tendencies, as this was beyond the scope of the present paper.

\section{Methods}

\section{Participants}

For study 2, 385 participants were recruited through Amazon Mechanical Turk (MTurk) online platform, and each participant was paid \$4.50. Prior to data analysis, 126 participants (32.7\%) were removed, in line with guidance from Meade and Craig (2012) due to: failure of one or both attention checks $(n=28)$, being identified as a bot via repeated answers in the AUT $(n=62)$, poor English proficiency identified by lack of understanding of the AUT through irrelevant or incoherent answers $(n=20)$, lack of effort operationalised as $\leq 1$ responses on the AUT $(n=5)$, repeat participation in the study identified via duplicated IP addresses ( $\mathrm{n}$ $=8$ ), and finally $\geq 1$ missing answers on the HTQ $(n=3) .259$ participants remained, consisting 
of $56 \%$ males, $43 \%$ females and $1 \%$ other/unspecified, between the ages of 19 and $73(\mathrm{M}=$ 37.372, $\mathrm{SD}=11.280$ ), all of whom were based in the United States of America. The sample population identified as $68.3 \%$ White, $13.5 \%$ Black or African American, 5.8\% Mixed ethnicity, 4.6\% Asian, 4.6\% Hispanic/Latino, 1.2\% American Indian or Alaska Native, 0.4\% Native American/Pacific Islander, $1.2 \%$ other, $0.4 \%$ unspecified. The highest stages of educational attainment of the sample were as follows: $0.4 \%$ had achieved less than a high school degree, $13.1 \%$ had graduated high school, $22.0 \%$ had completed some school but did not have a degree, $13.9 \%$ had completed a 2-year Associate degree in college, $43.2 \%$ had completed a 4-year Bachelor's degree in college, $6.6 \%$ had a Master's degree, and $0.8 \%$ had a Doctoral or Professional degree. Ethical approval for the study was acquired from the Department of Psychology Ethics Committee of the University of Cambridge. In line with the Declaration of Helsinki (1964), electronic informed consent was obtained from all participants before beginning the survey, and participants were notified that they may terminate their participation in the study at any point.

\section{Measures}

We administered the HTQ (Ramakrishnan, Robbins, \& Zmigrod, 2021), rated on 7point Likert scales ranging from "Strongly disagree" to "Strongly agree", along with the additional measures and cognitive tasks, in the form of an electronic survey hosted by Qualtrics Survey Software. Consistent with Study 1, measures consisted of the revised OCI (Foa et al., 2002), which had a high Cronbach's $\alpha$ value of 0.944, and the AUT (Guilford, 1967; Zmigrod et al., 2019), which had a high Cronbach's $\alpha$ value of 0.791, as well as the Creature of Habit Scale (Ersche et al., 2017), which had a high Cronbach's $\alpha$ value of 0.902 . The survey also included two interspersed attention checks, again as in Study 1. 


\section{$\underline{\text { Results }}$}

\section{Correlational analysis}

Similarly to Study 1 , in order to consider any confounding variables, we examined the correlations between the demographic variables and the psychological variables of interest. Age was found to be negatively correlated with Compulsivity $(r=-0.212, p<0.001)$ and the OCI $(r=-0.277, p<0.001)$, and positively correlated with Aversion to Novelty $(r=0.178, p$ $=0.004)$, AUT elaboration $(r=0.141, p<0.024)$ and AUT fluency $(r=0.141, p=0.024)$. Gender differences were present for Aversion to Novelty, $t(255)=-2.128, p=0.034$; and AUT fluency, $t(252)=-2.604, p=0.010$, with females scoring more highly than males in both Aversion to Novelty and AUT fluency. Educational attainment was not significantly correlated with any of the three subscales, AUT measures, or the OCI. Therefore, the demographic variables of age and gender were included as covariates in further analyses.

\section{Relationship between cognitive flexibility and subclinical OCD traits}

In order to evaluate the relationships between cognitive flexibility and subclinical OCD symptomatology, we computed the Pearson's correlations for these variables (see Table 4). As evident in Table 4 and Figure 3, there was a significant negative correlation between AUT Flexibility and the OCI $(r=-0.362, p<0.001)$, as hypothesised, suggesting that individuals with increased subclinical OCD traits have a tendency towards increased cognitive rigidity, or decreased cognitive flexibility. The Pearson's $r$ effect size of -0.362 is relatively large, as per the individual differences research guidelines set out by Gignac and Szodorai (2016).

To complement the Pearson's correlations, we also examined the Bayes Factors (see Table 4), which demonstrated that the relationship between AUT Flexibility and the OCI possesses an extremely large Bayes Factor of $2.193 \times 10^{6}$ (see Table 4), indicating that the observed data is $2.193 \times 10^{6}$ times more likely under $\mathrm{H}_{1}$ (significant correlation) than $\mathrm{H}_{0}$ (no 
correlation). As this Bayes Factor value is above 100, it indicates "extreme evidence" for $\mathrm{H}_{1}$, in line with the guidelines from Wagenmakers and colleagues (2018).

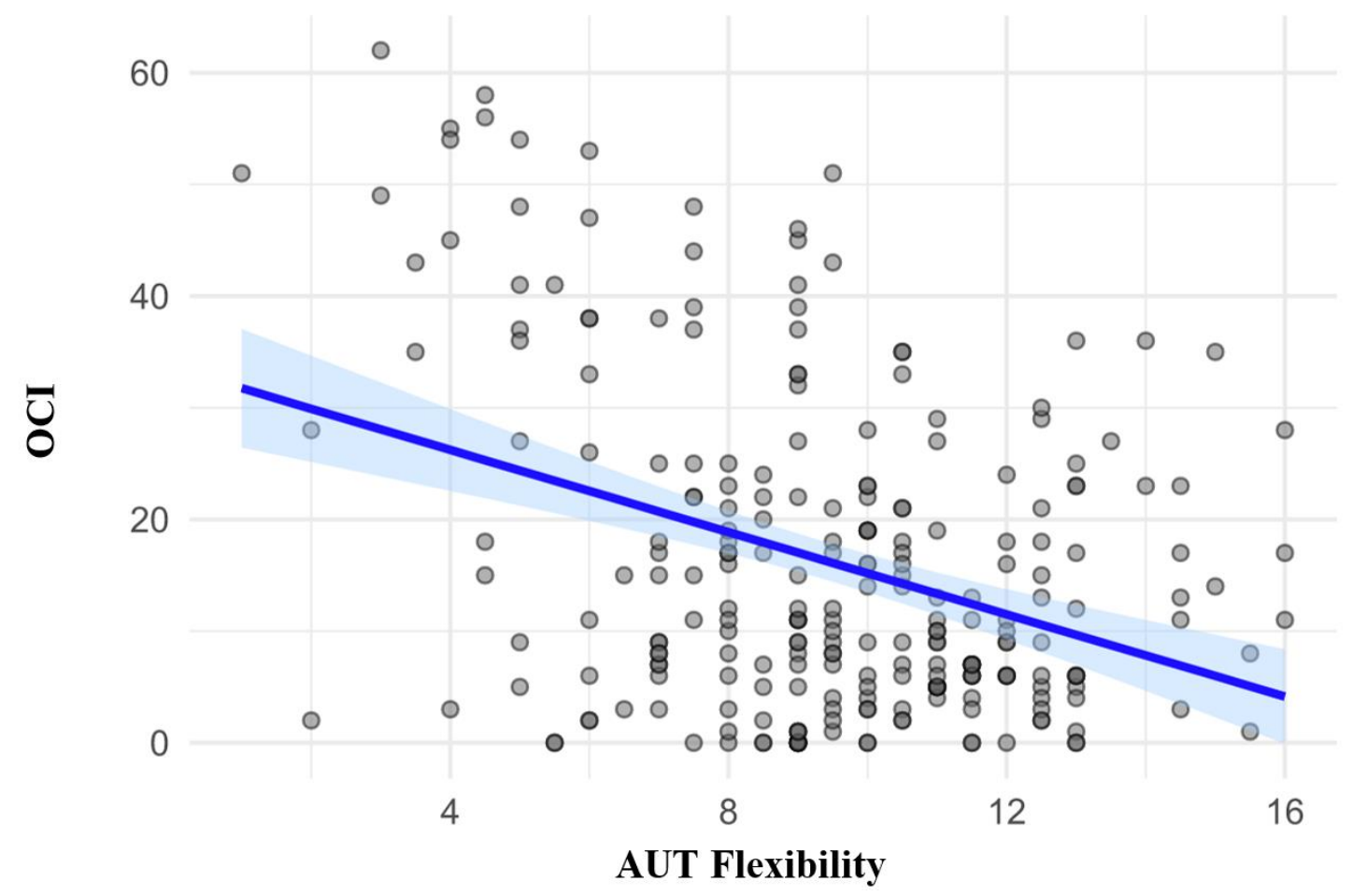

Figure 3. Scatter plot showing correlations between the Obsessive-Compulsive Inventory (OCI) and the Flexibility component of the Alternative Uses Task (AUT).

\section{Relationship between HTQ and subclinical OCD traits}

There was a significant positive correlation between habitual tendencies, measured by the HTQ, and subclinical OCD traits, measured by the OCI $(r=0.258, p<0.001)$. Of the three HTQ subscales, Compulsivity showed the strongest correlation with the OCI $(r=0.461, p<$ 0.001), and this relationship possessed an extremely large Bayes Factor of $5.094 \times 10^{11}$ (see Table 4), indicating that the observed data is $5.094 \times 10^{11}$ times more likely under $\mathrm{H}_{1}$ (significant correlation) than $\mathrm{H}_{0}$ (no correlation). 
Table 4. Correlation matrix of habitual tendencies, OCD traits and cognitive measures, including Pearson's correlations and Bayes Factors.

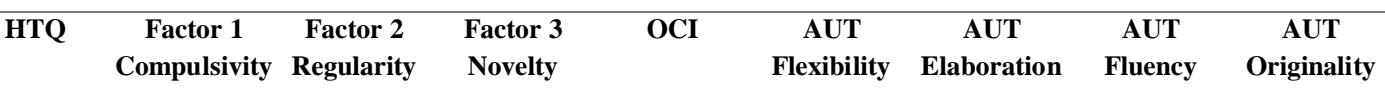

\begin{tabular}{|c|c|c|c|c|c|c|c|c|c|c|}
\hline \multirow[t]{2}{*}{ HTQ } & $\mathrm{r}$ & - & & & & & & & & \\
\hline & $\mathrm{BF}_{10}$ & - & & & & & & & & \\
\hline \multirow{2}{*}{$\begin{array}{l}\text { Factor } 1 \\
\text { Compulsivity }\end{array}$} & $\mathrm{r}$ & $0.747 * * *$ & - & & & & & & & \\
\hline & $\overline{\mathrm{BF}_{10}}$ & $1.188 \times 10^{44}$ & - & & & & & & & \\
\hline \multirow{2}{*}{$\begin{array}{l}\text { Factor } 2 \\
\text { Regularity }\end{array}$} & $\mathrm{r}$ & $0.782 * * *$ & $0.314 * * *$ & - & & & & & & \\
\hline & $\mathrm{BF}_{10}$ & $2.284 \times 10^{51}$ & 44224.531 & - & & & & & & \\
\hline \multirow[t]{2}{*}{ Factor 3 Novelty } & $\mathrm{r}$ & $0.685 * * *$ & $0.198 * *$ & $0.465 * * *$ & - & & & & & \\
\hline & $\mathrm{BF}_{10}$ & $8.676 \times 10^{33}$ & 12.917 & $2.355 \times 10^{12}$ & - & & & & & \\
\hline \multirow[t]{2}{*}{$\overline{\mathrm{OCI}}$} & $\mathrm{r}$ & $0.258 * * *$ & $0.461 * * *$ & 0.095 & -0.080 & - & & & & \\
\hline & $\mathrm{BF}_{10}$ & 404.171 & $5.094 \times 10^{11}$ & 0.240 & 0.173 & - & & & & \\
\hline \multirow{2}{*}{$\begin{array}{l}\text { AUT } \\
\text { Flexibility }\end{array}$} & $\mathrm{r}$ & -0.026 & -0.048 & -0.090 & 0.104 & $-0.362 * * *$ & - & & & \\
\hline & $\mathrm{BF}_{10}$ & 0.085 & 0.105 & 0.217 & 0.305 & $2.193 \times 10^{6}$ & - & & & \\
\hline \multirow[t]{2}{*}{ AUT Elaboration } & $\mathrm{r}$ & 0.045 & -0.002 & 0.035 & 0.082 & $-0.136 *$ & $0.414 * * *$ & - & & \\
\hline & $\mathrm{BF}_{10}$ & 0.101 & 0.078 & 0.092 & 0.184 & 0.774 & $1.516 \times 10^{9}$ & - & & \\
\hline \multirow{2}{*}{$\begin{array}{l}\text { AUT } \\
\text { Fluency }\end{array}$} & $\mathrm{r}$ & -0.074 & -0.093 & -0.104 & 0.055 & $\mathbf{- 0 . 3 2 6 * * *}$ & $0.867 * * *$ & $0.423 * * *$ & - & \\
\hline & $\mathrm{BF}_{10}$ & 0.156 & 0.233 & 0.307 & 0.115 & 70149.321 & $8.851 \times 10^{74}$ & $4.991 \times 10^{9}$ & - & \\
\hline \multirow[t]{2}{*}{ AUT Originality } & $\mathrm{r}$ & -0.058 & 0.026 & $-0.162 * *$ & -0.002 & $-0.172 * *$ & $0.617 * * *$ & $0.332 * * *$ & $0.668 * * *$ & - \\
\hline & $\mathrm{BF}_{10}$ & 0.119 & 0.085 & 2.199 & 0.078 & 3.072 & $1.336 \times 10^{25}$ & 184289.365 & $1.760 \times 10^{31}$ & - \\
\hline
\end{tabular}

${ }^{*} \mathrm{p}<0.05,{ }^{* *} \mathrm{p}<0.01,{ }^{* * *} \mathrm{p}<0.001 ; \mathrm{BF}<3=$ Anecdotal evidence; $\mathrm{BF}<10=$ Moderate evidence; $\mathrm{BF}<30=$ Strong evidence; $\mathrm{BF}<100=$ Very strong evidence; $\mathrm{BF}>100=$ Extremely strong evidence. HTQ $=$ Habitual Tendencies Questionnaire. AUT $=$ Alternative Uses Task. OCI $=$ ObsessiveCompulsive Inventory.

\section{Cognitive predictors of subclinical OCD symptomatology}

We then carried out a multiple regression with the three HTQ subscales and the four AUT components as predictors of subclinical OCD symptomatology (see Table 5). Both HTQ Compulsivity and AUT Flexibility emerged as significant predictors of the OCI. Higher Compulsivity and lower cognitive flexibility predicted greater levels of subclinical OCD traits. As shown in Table 5, HTQ Compulsivity and cognitive flexibility (as measured by the AUT) were significant and unique predictors of the OCI. In order to examine whether there was a significant interaction between these two predictors, hierarchical linear regression was then conducted (see Table 6). In Step 1, the demographic variables age and gender were entered as covariates. As shown in Table 6, age was a significant negative predictor of subclinical OCD 
symptomatology, such that older participants exhibited lower levels of subclinical OCD symptomatology than younger participants in the present sample. In Step 2, HTQ compulsivity and cognitive flexibility (as measured by the AUT) were added, both of which were significant predictors of subclinical OCD symptomatology. HTQ Compulsivity had a positive relationship with the OCI $(\beta=0.416, p<0.001)$ and cognitive flexibility had a negative relationship with the OCI $(\beta=-0.330, p<0.001)$. These independent variables accounted for a significant proportion of the variance in subclinical OCD symptomatology $\left(r^{2}=0.352\right)$. In Step 3 , the interaction term for HTQ Compulsivity and cognitive flexibility was entered. There was a significant interaction effect between compulsivity and cognitive flexibility, as shown in Table 6 , with $\beta=-0.706, p=0.004$. The interaction term increased the $r^{2}$ value to 0.373 , thus accounting for a further $2.1 \%$ of the variance in subclinical OCD symptomatology.

Table 5. Multiple regression with three Habitual Tendencies Questionnaire (HTQ) subscales and four Alternative Uses Task (AUT) components as predictors of subclinical OCD symptomatology (as measured by the Obsessive-Compulsive Inventory, OCI), with demographic variables age and gender as covariates

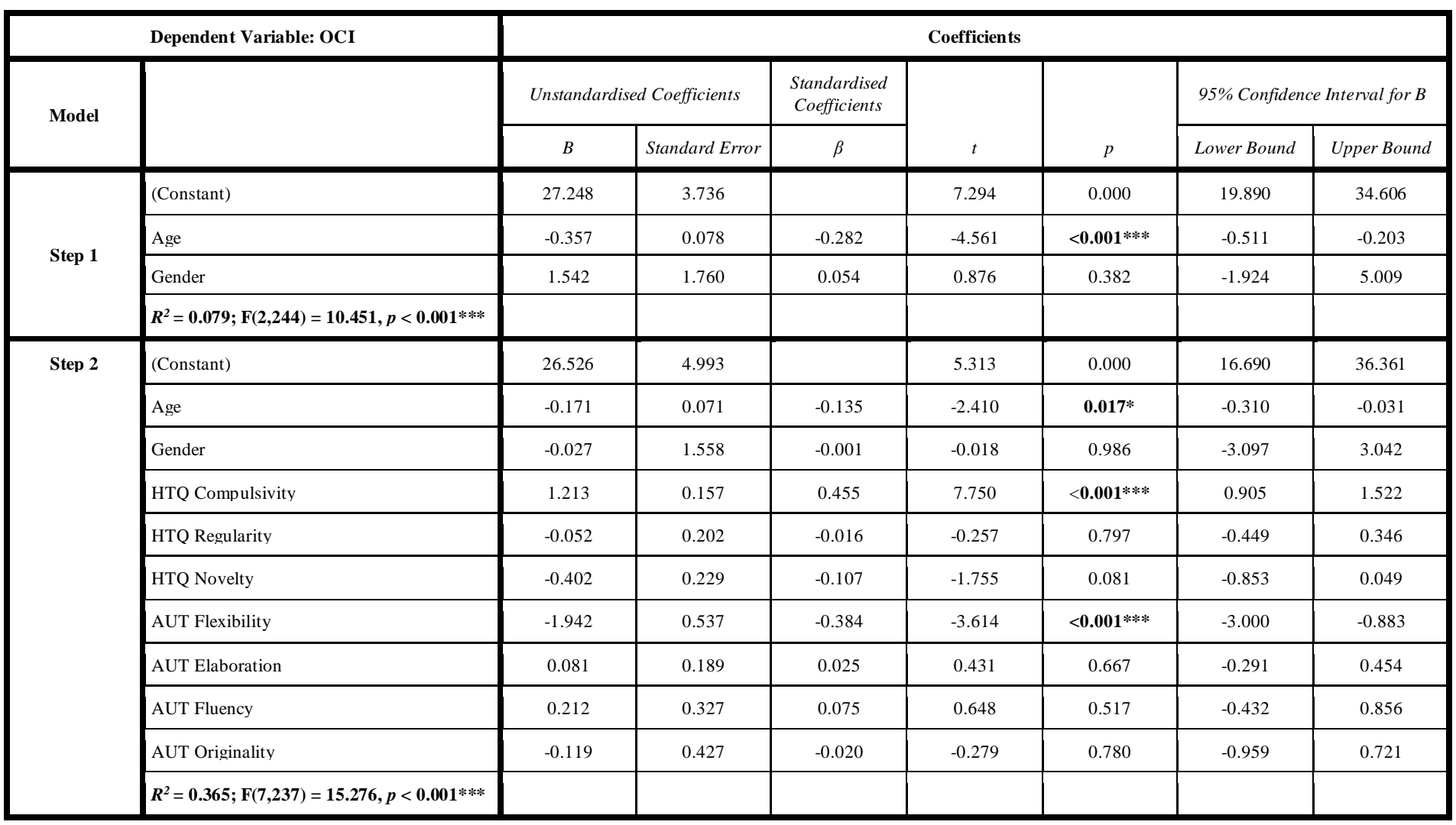


Table 6. 3-step hierarchical linear regression with the Compulsivity subscale of the Habitual Tendencies Questionnaire (HTQ), AUT (Alternative Uses Task) Flexibility and the interaction term between them as predictors of subclinical OCD symptomatology (as measured by the Obsessive-Compulsive Inventory, OCI), with demographic variables age and gender as covariates.

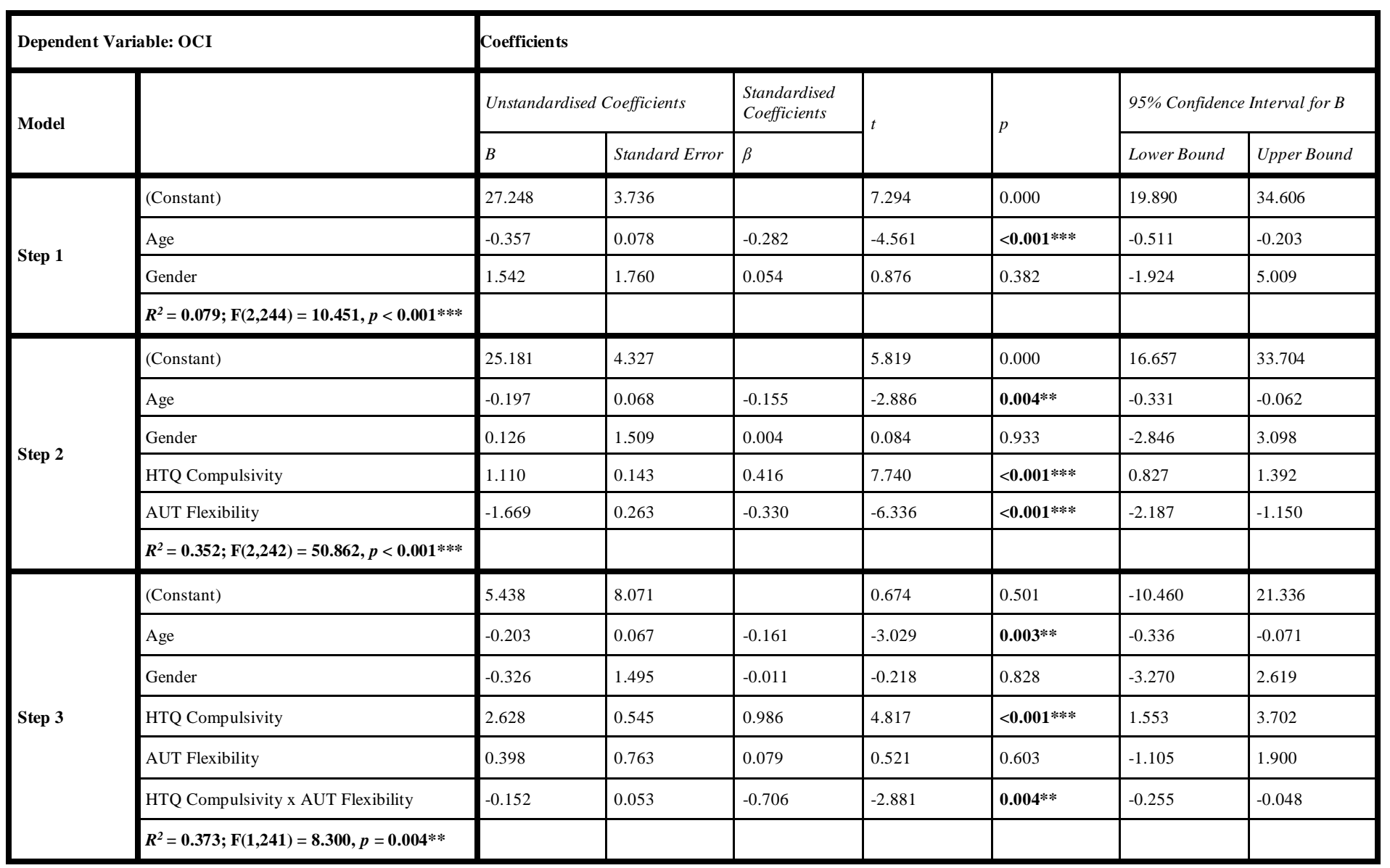

\section{Interaction effects between habitual compulsivity, cognitive flexibility and subclinical}

\section{OCD traits}

Simple slope analyses (SSA) were conducted to investigate the association between cognitive flexibility and subclinical OCD symptomatology at 1 standard deviation (SD) above and below mean HTQ Compulsivity, with age and gender as covariates (see Figure 4a). A significant negative relationship was found between cognitive flexibility and subclinical OCD symptomatology when HTQ Compulsivity was high (at $+1 \mathrm{SD}, b=-2.32, p<0.001$ ), while no significant relationship was found when HTQ Compulsivity was low (at $-1 \mathrm{SD}, b=-0.68, p=$ 
0.11). Carrying out the reciprocal SSA to investigate the association between HTQ Compulsivity and subclinical OCD symptomatology (see Figure 4b) demonstrated that there were significant positive relationships between HTQ Compulsivity and subclinical OCD symptomatology both when cognitive flexibility was high (at $+1 \mathrm{SD}, b=0.76, p<0.001$ ), and when it was low (at $-1 \mathrm{SD}, b=1.62, p<0.001$ ). The interaction effects between HTQ Compulsivity and cognitive flexibility (measured by the AUT) are shown in the filled contour plot in Figure 4c. This shows that the relationship between HTQ Compulsivity and subclinical OCD symptomatology varies depending on cognitive flexibility, such that at high levels but not low levels of HTQ Compulsivity, cognitive flexibility differentiates between high and low levels of subclinical OCD symptomatology. It also shows that the relationship between cognitive flexibility and subclinical OCD symptomatology varies depending on HTQ Compulsivity, such that at both high and low levels of cognitive flexibility, HTQ Compulsivity differentiates between high and low levels of subclinical OCD symptomatology. The highest levels of subclinical OCD traits were observed in participants with high HTQ Compulsivity scores and low AUT Flexibility scores, indicating a compensatory effect, in accordance with the significant interaction effect shown in the hierarchical linear regression (see Table 6). Meanwhile, the lowest levels of subclinical OCD traits were observed in participants with low HTQ Compulsivity scores, regardless of their AUT Flexibility scores. Therefore, high HTQ Compulsivity and low cognitive flexibility are necessary for the highest levels of subclinical OCD symptomatology, while neither is sufficient independently. These findings are in line with those from the SSA analyses. We used the Johnson-Neyman technique to analyse this interaction further (Johnson and Neyman, 1936), which indicated that the association between cognitive flexibility and OCD traits was significantly negative at compulsivity scores of 8.12 and above (see Figure 4d). 
a)

छ

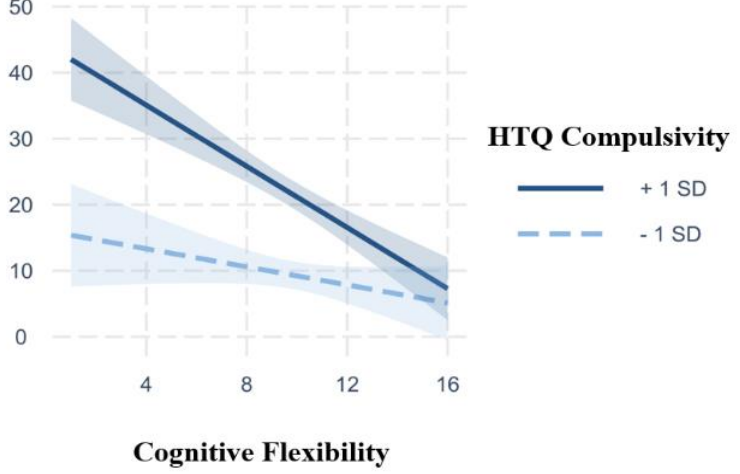

c)

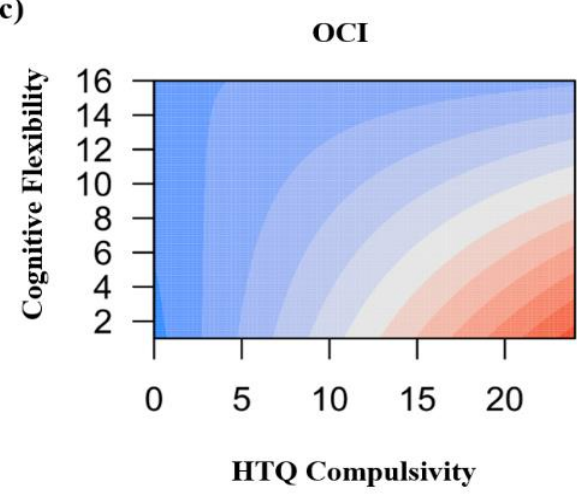

b)

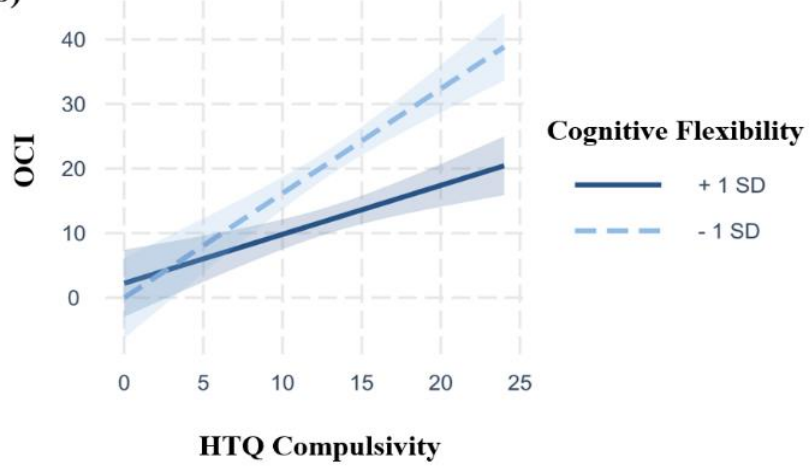

d)

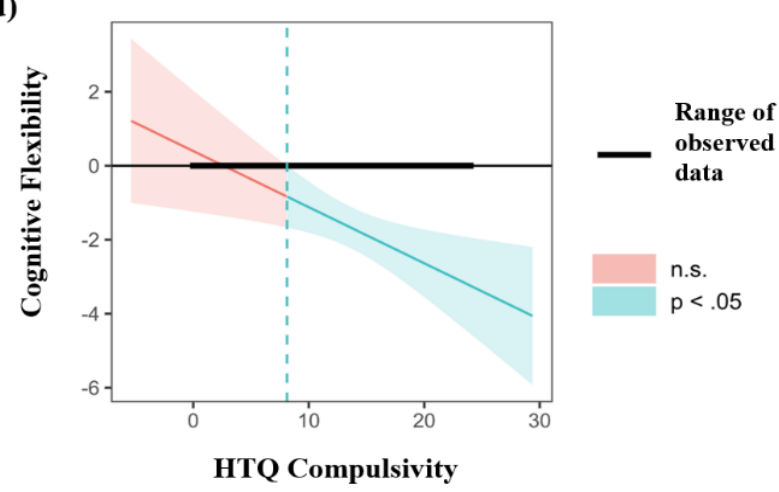

Figure 4. a) Interaction plot between the Compulsivity subscale of the Habitual Tendencies Questionnaire (HTQ), cognitive flexibility (as measured by the Alternative Uses Task) and subclinical OCD symptomatology (as measured by the Obsessive-Compulsive Inventory, OCI) at $1 \mathrm{SD}$ above and below the mean, controlling for age and gender, with cognitive flexibility as the predictor and HTQ Compulsivity as the moderator. Created using the interactions and interplot packages in the statistical software $\mathrm{R}$ Studio. b) Interaction plot between the Compulsivity subscale of the Habitual Tendencies Questionnaire (HTQ), cognitive flexibility (as measured by the Alternative Uses Task) and subclinical OCD symptomatology (as measured by the Obsessive-Compulsive Inventory, OCI) at 1 SD above and below the mean, controlling for age and gender, with HTQ Compulsivity as the predictor and cognitive flexibility as the moderator. c) Representation of the regression surface predicting subclinical OCD symptomatology (as measured by the Obsessive-Compulsive Inventory, OCI) as a function of the Compulsivity subscale of the Habitual Tendencies Questionnaire (HTQ) and cognitive flexibility (as measured by the Alternative Uses Task), while controlling for age and gender. (Created using the visreg package in the statistical software R Studio.) d) JohnsonNeyman plot showing the conditional relation between cognitive flexibility and OCD symptomatology as a function of the Compulsivity subscale of the Habitual Tendencies Questionnaire (HTQ). The solid diagonal line represents the regression coefficient of cognitive flexibility (as measured by the Alternative Uses Task) for OCD symptomatology along the compulsivity spectrum. The dashed vertical line at a HTQ Compulsivity value of 8.12 represents the transition from significance to non-significance. The width of the regions reflects the $95 \%$ confidence intervals. (Created using the interactions and interplot packages in the statistical software R Studio.) 


\section{Discussion}

The present study has demonstrated that behaviourally-assessed cognitive inflexibility predicts high levels of OCD traits in the general population, and that cognitive inflexibility interacts with habitual compulsivity to predict the highest severity of OCD symptoms. Notably, both rigidity and habitual compulsivity are necessary, and neither is sufficient alone for high OCD symptoms. Thus, in line with our first hypothesis, $\boldsymbol{H 1}$, individuals with lower cognitive flexibility showed increased subclinical OCD symptomatology. In line with our second hypothesis, $\boldsymbol{H} \mathbf{2}$, cognitive inflexibility interacted with habitual compulsivity to account for $49.4 \%$ of the variance in subclinical OCD symptomatology in Study 1, and 37.3\% in Study 2, demonstrating reproducibility across two independent samples. Simple slope analyses demonstrated that cognitive inflexibility differentiated between high and low levels of OCD traits when habitual compulsivity was high, but not when it was low, suggesting that habitual compulsivity moderates the negative relationship between cognitive flexibility and subclinical OCD symptomatology. Furthermore, the highest levels of OCD traits were seen in participants with high HTQ Compulsivity scores and low AUT Flexibility scores, suggesting a compensatory effect (see Figures $2 \mathrm{c}$ and $4 \mathrm{c}$ ).

The present study demonstrated cognitive rigidity to be a significant independent predictor of OCD traits. This provides support for cognitive flexibility accounts of OCD, and suggests that impaired cognitive flexibility may be one of the core cognitive underpinnings of OCD. Across the literature, there is marked variation in methods of measuring cognitive flexibility, and this may help to explain the heterogeneity of the findings concerning impaired cognitive flexibility in OCD. Cognitive flexibility is a complex multidimensional construct that has been subdivided in a number of different ways. For example, it may be broken down according to the various tasks used to measure it, including set shifting, reversal learning, cued task switching, and cognitive or motor inhibition tasks (Gruner \& Pittenger, 2017). Kanen and 
colleagues (2019) further subdivided one of these tasks, and demonstrated that a subcomponent of probabilistic reversal learning was increased in individuals with substance use disorder, but decreased in individuals with OCD, relative to controls. Similarly, a meta-analysis by Chamberlain and colleagues (2021) found that extra-dimensional, but not intra-dimensional, set-shifting is consistently impaired in OCD. These findings suggest that not all aspects of cognitive flexibility may be impaired in OCD. The difficulty with which rule changes may be detected, and thus the levels of feedback processing required, varies across the wide range of tasks used to measure cognitive flexibility (Fradkin et al., 2018). Therefore, deficits in feedback processing may, in part, account for the cognitive inflexibility found in OCD (Fradkin et al., 2018). The use of self-directed tasks to measure cognitive flexibility, such as the AUT used in the present study, may eliminate the confounding effects of feedback processing, as the shifting is internally generated. Therefore, these tasks may be able to specifically target the subdimensions of cognitive flexibility impaired in OCD..

The subdivision of cognitive flexibility into its constituent parts may be an important future direction in gaining more specific insight into the cognitive processes underlying OCD, as well as the heterogeneity of OCD symptoms (Bragdon et al., 2018). Indeed, another explanation for the highly mixed nature of the literature regarding the cognitive deficits associated with OCD may be the wide range of its clinical presentations. In a meta-analysis by Bragdon and colleagues (2018), increased symmetry and ordering symptoms were associated with reduced cognitive flexibility (measured using a mixture of externally-cued and selfdirected tasks across the included studies) and verbal working memory, while increased obsessing and checking symptoms were associated with poorer memory and verbal memory. Therefore, in addition to the subdivision of constructs such as cognitive flexibility, it may also be important to separate OCD into its different symptom groups, in order to gain more specific insight into its cognitive underpinnings. 
Furthermore, the moderation of cognitive flexibility by habitual tendencies adds nuance to the habit hypothesis of OCD proposed by Graybiel and Rauch (2000), suggesting that cognitive flexibility must be studied along with habitual tendencies, thus building on previous contributions to this debate. For example, Gillan and colleagues (2015) found that excessive habits in OCD patients were associated with hyperactivation in the caudate nucleus, an important region for goal-directed behaviour that is implicated in the pathophysiology of OCD. Therefore, habit-forming biases in OCD may be a result of impairments in this neural circuitry. Another study by Gillan and colleagues (2011) used outcome devaluation paradigms to demonstrate increased habitual tendencies in OCD patients. As the tasks used in Gillan and colleagues' (2011) study did not involve obsessions, it has been suggested that the tendency towards developing compulsive-like habits in OCD is independent of obsessions, and thus compulsions are a core component of OCD (Gillan and Sahakian, 2015). Gillan and colleagues (2014) further found that individuals with OCD showed increased habitual responding in a shock-avoidance paradigm, and when trying to explain their behaviour, erroneously deduced that if they felt compelled to perform the habitual response, then there must be something to fear. In contrast with the majority of the preceding literature on $\mathrm{OCD}$, this reverse inference suggests that obsessions may in fact arise as a result of compulsions. This provides further evidence for the habit hypothesis of OCD, and emphasises the importance of studying compulsivity in relation to habits and OCD. The HTQ, and in particular its Compulsivity subscale, may be valuable as a predictive or screening measure for OCD traits (Ramakrishnan, Robbins \& Zmigrod, 2021). The present study extends the potential applications of the HTQ, such that it may be used in conjunction with measures of cognitive flexibility as an even stronger predictor of subclinical OCD symptomatology.

The moderation of cognitive flexibility by habitual tendencies suggests that neither construct alone is sufficient to explain the roots of OCD, but that the interactions between the 
two must be studied in order to develop more comprehensive theories. A recent review conducted by Fullana and colleagues (2020) was unable to identify any biomarkers with diagnostic specificity for OCD. However, as biomarkers are only one small aspect of the RDoC framework, perhaps more integrative $\mathrm{RDoC}$ approaches using combinations of biomarkers and their interactions with other constructs may provide a solution. In addition, future use of interactionist, individual differences approaches may help to explain past empirical inconsistencies regarding excessive cognitive inflexibility and habitual tendencies in OCD (e.g. Kalanthroff et al., 2020; Shin et al., 2014; Snyder et al., 2015).

The importance of studying individual differences in cognitive flexibility, habitual tendencies, and the interactions between them in order to elucidate the cognitive processes underlying OCD is emphasised by neurobiological findings. Vaghi and colleagues (2017) discovered that reduced functional connectivity between the caudate and the ventrolateral prefrontal cortex was selectively associated with reduced cognitive flexibility, while reduced functional connectivity between the putamen and the dorsolateral prefrontal cortex was selectively associated with goal-directed performance, as well as OCD symptom severity. Another double dissociation was found by Tyagi and colleagues (2019), who showed that anteromedial subthalamic nucleus deep brain stimulation in patients with treatment refractory OCD selectively improved cognitive flexibility, while ventral capsule/ventral striatal deep brain stimulation selectively improved mood. Deep brain stimulation at each site independently reduced OCD symptoms. The above neurobiological findings are reflected in the integrated model of OCD proposed by Robbins and colleagues (2019), and corroborate the importance of the three main factors in OCD of varying severity, namely cognitive inflexibility; habitual rather than goal-directed behaviour; and mood. Furthermore, these neurobiological findings have potential therapeutic applications for those suffering from severe, treatment-refractory OCD. 
In order to ensure that the present study was well-powered to detect the present effects, we conducted a conservative post-hoc power analysis $(\alpha=0.001, \mathrm{n}=130$ for Study $1 ; \mathrm{n}=259$ for Study 2), using the pwr package in the statistical software R Studio. For the effect sizes, we used the smallest relevant Pearson's correlations: 0.390 for Study 1 and 0.362 for Study 2, which reflected the correlations between AUT flexibility and the OCI (see Tables 1 and 4). This revealed power of 0.912 for Study 1 and 0.997 for Study 2, indicating that the sample sizes were sufficient. Future studies using even larger sample sizes would help to replicate and extend the present findings. The present investigation furthers our understanding of the cognitive underpinnings of obsessive-compulsive symptoms, illustrating their interactive underpinnings in rigidity and habitual tendencies. Replication of these findings in children and adolescents would be useful in considering developmental trajectories and in exploring whether early compulsivity or cognitive rigidity can help to predict the development of OCD in later life. This could have important implications in the development of early interventions for OCD. Notably, adolescents with OCD do not show deficits in performance on the Wisconsin Card Sort Test, an externally-cued measure of cognitive flexibility (Marzuki et al., submitted), and as such, an important future direction would be to administer a self-directed measure of cognitive flexibility, such as the AUT, in this age group. In order to explore whether the present findings are consistent across cultural contexts, cross-cultural replication is imperative. Interestingly, cognitive rigidity may also account for the finding of heightened moral rigidity in OCD (Whitton et al., 2014). Furthermore, impaired cognitive flexibility is not a characteristic limited to OCD, but has been demonstrated in other disorders, such as binge eating disorder (Duchesne et al., 2010) and addictions (Verdejo-Garcia et al., 2015). Therefore, it is important to explore whether habitual tendencies, especially compulsivity, interact with cognitive inflexibility to predict symptom severity in these other conditions, or whether this interaction effect is specific to OCD. 
The present study is not without its limitations. Firstly, the use of an online convenience sample may not be representative of the general population, and so future research may administer the study in a laboratory setting or a population sampled specifically for representativeness. Secondly, the present findings are in a healthy population with subclinical OCD symptoms, and thus replication in patients with clinical OCD and OCPD is vital. Finally, the present study focuses on one aspect of the cognitive flexibility construct, by using the AUT. In order to fully characterise the cognitive flexibility construct, future research must deconstruct it, by including additional measures of cognitive flexibility, such as the Wisconsin Card Sort Test (Berg, 1948), or the Intra-Dimensional Extra-Dimensional set-shift CANTAB task (Downes et al., 1989).

To conclude, the present study demonstrated that both cognitive flexibility and habitual compulsivity acted as independent significant predictors of subclinical OCD symptomatology, but also exhibited an important interaction. The interaction between cognitive flexibility and habitual compulsivity accounted for almost half of the variance in subclinical OCD symptomatology. Furthermore, both cognitive inflexibility and habitual compulsivity are necessary for high levels of OCD symptoms, while neither is sufficient alone. These findings may prove useful for future research into both subclinical and clinical OCD traits, as well as other disorders involving cognitive inflexibility. The results may also help in the development of interventions targeting the impaired cognitive flexibility and maladaptive habits proposed to underlie OCD, thus helping to alleviate the debilitating symptoms that cause so many disruptions and difficulties in patients' daily lives. 


\section{References}

Abramovitch, A., \& Cooperman, A. (2015). The cognitive neuropsychology of obsessivecompulsive disorder: A critical review. Journal of Obsessive-Compulsive and Related Disorders, 5, 24-36.

Abramovitch, A., De Nadai, A. S., \& Geller, D. A. (2021). Neurocognitive endophenotypes in pediatric OCD probands, their unaffected parents and siblings. Progress in NeuroPsychopharmacology and Biological Psychiatry, 110, 110283.

Armitage, C. J., \& Christian, J. (2003). From attitudes to behaviour: Basic and applied research on the theory of planned behaviour. Current Psychology, 22(3), 187-195.

Bannon, S., Gonsalvez, C. J., Croft, R. J., \& Boyce, P. M. (2006). Executive functions in obsessive-compulsive disorder: state or trait deficits?. Australian \& New Zealand Journal of Psychiatry, 40(11-12), 1031-1038.

Bari, A., \& Robbins, T. W. (2013). Inhibition and impulsivity: behavioral and neural basis of response control. Progress in neurobiology, 108, 44-79.

Bar-Tal, Y. (1994). The effect on mundane decision-making of the need and ability to achieve cognitive structure. European Journal of Personality, 8(1), 45-58.

Benton, A. L., Hamsher, K., \& Sivan, A. B. (1983). Controlled oral word association test (COWAT). Multilingual Aphasia Examination, 3rd ed. Iowa City, IA: AJA Associates.

Berg, E. A. (1948). A simple objective technique for measuring flexibility in thinking. The Journal of general psychology, 39(1), 15-22. 
Bragdon, L. B., Gibb, B. E., \& Coles, M. E. (2018). Does neuropsychological performance in OCD relate to different symptoms? A meta-analysis comparing the symmetry and obsessing dimensions. Depression and anxiety, 35(8), 761-774.

Caudek, C., Sica, C., Marchetti, I., Colpizzi, I., \& Stendardi, D. (2020). Cognitive inflexibility specificity for individuals with high levels of obsessive-compulsive symptoms. Journal of Behavioral and Cognitive Therapy, 30(2), 103-113.

Ceaser, A. E., Goldberg, T. E., Egan, M. F., McMahon, R. P., Weinberger, D. R., \& Gold, J. M. (2008). Set-shifting ability and schizophrenia: a marker of clinical illness or an intermediate phenotype?. Biological psychiatry, 64(9), 782-788.

Chamberlain, S. R., Blackwell, A. D., Fineberg, N. A., Robbins, T. W., \& Sahakian, B. J. (2005). The neuropsychology of obsessive compulsive disorder: the importance of failures in cognitive and behavioural inhibition as candidate endophenotypic markers. Neuroscience \& Biobehavioral Reviews, 29(3), 399-419.

Chamberlain, S. R., Fineberg, N. A., Blackwell, A. D., Robbins, T. W., \& Sahakian, B. J. (2006). Motor inhibition and cognitive flexibility in obsessive-compulsive disorder and trichotillomania. American Journal of Psychiatry, 163(7), 1282-1284.

Chamberlain, S. R., Fineberg, N. A., Menzies, L. A., Blackwell, A. D., Bullmore, E. T., Robbins, T. W., \& Sahakian, B. J. (2007). Impaired cognitive flexibility and motor inhibition in unaffected first-degree relatives of patients with obsessive-compulsive disorder. American Journal of Psychiatry, 164(2), 335-338.

Chamberlain, S. R., Solly, J. E., Hook, R. W., Vaghi, M. M., \& Robbins, T. W. (2021). Cognitive Inflexibility in OCD and Related Disorders. Current Topics in Behavioral Neurosciences. 
Cheung, J. H., Burns, D. K., Sinclair, R. R., \& Sliter, M. (2017). Amazon Mechanical Turk in Organizational Psychology: An Evaluation and Practical Recommendations. Journal of Business and Psychology, 32(4), 347-361. https://doi.org/10.1007/s10869-016-9458-5

Dajani, D. R., \& Uddin, L. Q. (2015). Demystifying cognitive flexibility: implications for clinical and developmental neuroscience. Trends in neurosciences, 38(9), 571-578.

De Wit, S., Niry, D., Wariyar, R., Aitken, M. R. F., \& Dickinson, A. (2007). Stimulus-outcome interactions during instrumental discrimination learning by rats and humans. Journal of Experimental Psychology: Animal Behavior Processes, 33(1), 1.

Diedrich, A., \& Voderholzer, U. (2015). Obsessive-compulsive personality disorder: a current review. Current psychiatry reports, 17(2), 2.

Downes, J. J., Roberts, A. C., Sahakian, B. J., Evenden, J. L., Morris, R. G., \& Robbins, T. W. (1989). Impaired extra-dimensional shift performance in medicated and unmedicated Parkinson's disease: evidence for a specific attentional dysfunction. Neuropsychologia, 27(1112), 1329-1343.

Duchesne, M., Mattos, P., Appolinário, J. C., Freitas, S. R. D., Coutinho, G., Santos, C., \& Coutinho, W. (2010). Assessment of executive functions in obese individuals with binge eating disorder. Brazilian Journal of Psychiatry, 32(4), 381-388.

Eisen, J. L., Mancebo, M. A., Pinto, A., Coles, M. E., Pagano, M. E., Stout, R., \& Rasmussen, S. A. (2006). Impact of obsessive-compulsive disorder on quality of life. Comprehensive psychiatry, 47(4), 270-275.

Ersche, K. D., Lim, T. V., Ward, L. H., Robbins, T. W., \& Stochl, J. (2017). Creature of Habit: A self-report measure of habitual routines and automatic tendencies in everyday life. Personality and individual differences, 116, 73-85. 
Eslinger, P. J., \& Grattan, L. M. (1993). Frontal lobe and frontal-striatal substrates for different forms of human cognitive flexibility. Neuropsychologia, 31(1), 17-28.

Fineberg, N. A., Day, G. A., de Koenigswarter, N., Reghunandanan, S., Kolli, S., JefferiesSewell, K., ... \& Laws, K. R. (2015). The neuropsychology of obsessive-compulsive personality disorder: a new analysis. CNS spectrums, 20(5), 490-499.

Foa, E. B., Huppert, J. D., Leiberg, S., Langner, R., Kichic, R., Hajcak, G., \& Salkovskis, P. M. (2002). The Obsessive-Compulsive Inventory: development and validation of a short version. Psychological assessment, 14(4), 485.

Fradkin, I., Strauss, A. Y., Pereg, M., \& Huppert, J. D. (2018). Rigidly applied rules? Revisiting inflexibility in obsessive compulsive disorder using multilevel meta-analysis. Clinical Psychological Science, 6(4), 481-505.

Frenkel-Brunswik, E. (1949). Intolerance of ambiguity as an emotional and perceptual personality variable. Journal of personality, 18(1), 108-143.

Fullana, M. A., Abramovitch, A., Via, E., López-Sola, C., Goldberg, X., Reina, N., ... \& Radua, J. (2020). Diagnostic biomarkers for obsessive-compulsive disorder: A reasonable quest or ignis fatuus?. Neuroscience \& Biobehavioral Reviews.

Gignac, G. E., \& Szodorai, E. T. (2016). Effect size guidelines for individual differences researchers. Personality and individual differences, 102, 74-78.

Gillan, C. M., Papmeyer, M., Morein-Zamir, S., Sahakian, B. J., Fineberg, N. A., Robbins, T. W., \& de Wit, S. (2011). Disruption in the balance between goal-directed behavior and habit learning in obsessive-compulsive disorder. American Journal of Psychiatry, 168(7), 718-726. 
Gillan, C. M., Morein-Zamir, S., Urcelay, G. P., Sule, A., Voon, V., Apergis-Schoute, A. M., ... \& Robbins, T. W. (2014). Enhanced avoidance habits in obsessive-compulsive disorder. Biological psychiatry, 75(8), 631-638.

Gillan, C. M., \& Robbins, T. W. (2014). Goal-directed learning and obsessive-compulsive disorder. Philosophical Transactions of the Royal Society B: Biological Sciences, 369(1655), 20130475.

Gillan, C. M., Apergis-Schoute, A. M., Morein-Zamir, S., Urcelay, G. P., Sule, A., Fineberg, N. A., ... \& Robbins, T. W. (2015). Functional neuroimaging of avoidance habits in obsessivecompulsive disorder. American Journal of Psychiatry, 172(3), 284-293.

Gillan, C. M., \& Sahakian, B. J. (2015). Which is the driver, the obsessions or the compulsions, in OCD?. Neuropsychopharmacology, 40(1), 247.

Gillan, C. M., Robbins, T. W., Sahakian, B. J., van den Heuvel, O. A., \& van Wingen, G. (2016). The role of habit in compulsivity. European Neuropsychopharmacology, 26(5), 828840.

Graybiel, A. M., \& Rauch, S. L. (2000). Toward a neurobiology of obsessive-compulsive disorder. Neuron, 28(2), 343-347.

Gruner, P., \& Pittenger, C. (2017). Cognitive inflexibility in obsessive-compulsive disorder. Neuroscience, 345, 243-255.

Gu, B. M., Park, J. Y., Kang, D. H., Lee, S. J., Yoo, S. Y., Jo, H. J., ... \& Kwon, J. S. (2008). Neural correlates of cognitive inflexibility during task-switching in obsessive-compulsive disorder. Brain, 131(1), 155-164.

Guilford, J. P. (1967). The nature of human intelligence. 
IBM Corp. Released 2020. IBM SPSS Statistics for Windows, Version 26.0. Armonk, NY: IBM Corp

IBM Corp. Released 2020. IBM SPSS Statistics for Windows, Version 27.0. Armonk, NY: IBM Corp

Jalal, B., Brühl, A., O’Callaghan, C., Piercy, T., Cardinal, R. N., Ramachandran, V. S., \& Sahakian, B. J. (2018). Novel smartphone interventions improve cognitive flexibility and obsessive-compulsive disorder symptoms in individuals with contamination fears. Scientific reports, $8(1), 1-11$.

JASP Team (2020). JASP (Version 0.12) [Computer software].

Jeffreys, H. (1961). Theory of probability, Clarendon.

Kalanthroff, E., Marsh, R., Hassin, R. R., \& Simpson, H. B. (2020). Evidence for trial-by-trial dynamic adjustment of task control in unmedicated adults with OCD. Behaviour research and therapy, 126, 103572.

Kanen, J. W., Ersche, K. D., Fineberg, N. A., Robbins, T. W., \& Cardinal, R. N. (2019). Computational modelling reveals contrasting effects on reinforcement learning and cognitive flexibility in stimulant use disorder and obsessive-compulsive disorder: remediating effects of dopaminergic D2/3 receptor agents. Psychopharmacology, 236(8), 2337-2358.

Kruglanski, A. W. (1990). Motivations for judging and knowing: Implications for causal attribution.

Krumrei-Mancuso, E. J., \& Rouse, S. V. (2016). The development and validation of the comprehensive intellectual humility scale. Journal of Personality Assessment, 98(2), 209-221. 
Lally, P., \& Gardner, B. (2013). Promoting habit formation. Health Psychology Review, 7(sup1), S137-S158.

Leyfer, O. T., Folstein, S. E., Bacalman, S., Davis, N. O., Dinh, E., Morgan, J., ... \& Lainhart, J. E. (2006). Comorbid psychiatric disorders in children with autism: interview development and rates of disorders. Journal of autism and developmental disorders, 36(7), 849-861.

Mac Donald Jr, A. P. (1970). Revised scale for ambiguity tolerance: Reliability and validity. Psychological reports, 26(3), 791-798.

Marzuki, A. A, Tomić, I., Ip, H. Y. S., Kanen, J. W., Gottwald, J., Kaser, M., Sule, A., ConwayMorris, A.,. Sahakian, B. J., Robbins, T.W. (submitted). Environmental stochasticity promotes altered latent decision-making in adolescents with OCD: A Computational Psychiatry Approach.

Meade, A. W., \& Craig, S. B. (2012). Identifying careless responses in survey data. Psychological Methods, 17(3), 437.

Meiran, N., Diamond, G. M., Toder, D., \& Nemets, B. (2011). Cognitive rigidity in unipolar depression and obsessive compulsive disorder: Examination of task switching, Stroop, working memory updating and post-conflict adaptation. Psychiatry research, 185(1-2), 149-156.

Menzies, L., Achard, S., Chamberlain, S. R., Fineberg, N., Chen, C. H., Del Campo, N., ... \& Bullmore, E. (2007). Neurocognitive endophenotypes of obsessive-compulsive disorder. Brain, 130(12), 3223-3236.

OCD UK. (2021). Occurrences of OCD. https://www.ocduk.org/ocd/how-common-is-ocd

Paast, N., Khosravi, Z., Memari, A. H., Shayestehfar, M., \& Arbabi, M. (2016). Comparison of cognitive flexibility and planning ability in patients with obsessive compulsive disorder, 
patients with obsessive compulsive personality disorder, and healthy controls. Shanghai archives of psychiatry, 28(1), 28.

Ramakrishnan, S., Robbins, T.W., \& Zmigrod, L. (2021). The Habitual Tendencies Questionnaire: A Tool for Psychometric Individual Differences Research. Personality and Mental Health. 1-17.

Robbins, T. W., Gillan, C. M., Smith, D. G., de Wit, S., \& Ersche, K. D. (2012). Neurocognitive endophenotypes of impulsivity and compulsivity: towards dimensional psychiatry. Trends in cognitive sciences, 16(1), 81-91.

Robbins, T. W., \& Costa, R. M. (2017). Habits. Current Biology, 27(22), R1200-R1206.

Robbins, T. W., Vaghi, M. M., \& Banca, P. (2019). Obsessive-compulsive disorder: puzzles and prospects. Neuron, 102(1), 27-47.

Rosa-Alcázar, Á., Olivares-Olivares, P. J., Martínez-Esparza, I. C., Parada-Navas, J. L., RosaAlcázar, A. I., \& Olivares-Rodríguez, J. (2020). Cognitive flexibility and response inhibition in patients with Obsessive-Compulsive Disorder and Generalized Anxiety Disorder. International Journal of Clinical and Health Psychology, 20(1), 20-28.

RStudio Team (2020). RStudio: Integrated Development for R. RStudio, Inc., Boston, MA URL http://www.rstudio.com/.

Shin, N. Y., Lee, T. Y., Kim, E., \& Kwon, J. S. (2014). Cognitive functioning in obsessivecompulsive disorder: a meta-analysis. Psychological medicine, 44(6), 1121.

Snyder, H. R., Kaiser, R. H., Warren, S. L., \& Heller, W. (2015). Obsessive-compulsive disorder is associated with broad impairments in executive function: A meta-analysis. Clinical Psychological Science, 3(2), 301-330. 
Stanley Budner, N. Y. (1962). Intolerance of ambiguity as a personality variable 1. Journal of personality, 30(1), 29-50.

Sternheim, L., van der Burgh, M., Berkhout, L. J., Dekker, M. R., \& Ruiter, C. (2014). Poor cognitive flexibility, and the experience thereof, in a subclinical sample of female students with obsessive-compulsive symptoms. Scandinavian journal of psychology, 55(6), 573-577.

Thompson, B. (2007). Factor analysis. The Blackwell Encyclopedia of Sociology.

Tyagi, H., Apergis-Schoute, A. M., Akram, H., Foltynie, T., Limousin, P., Drummond, L. M., ... \& Sahakian, B. J. (2019). A Randomised Trial Directly Comparing Ventral Capsule and Anteromedial Subthalamic Nucleus Stimulation in Obsessive Compulsive Disorder: Clinical and Imaging Evidence for Dissociable Effects. Biological Psychiatry.

Tomer, R., Fisher, T., Giladi, N., \& Aharon-Peretz, J. (2002). Dissociation between spontaneous and reactive flexibility in early Parkinson's disease. Cognitive and Behavioral Neurology, 15(2), 106-112.

Vaghi, M. M., Vértes, P. E., Kitzbichler, M. G., Apergis-Schoute, A. M., van der Flier, F. E., Fineberg, N. A., ... \& Bullmore, E. T. (2017). Specific frontostriatal circuits for impaired cognitive flexibility and goal-directed planning in obsessive-compulsive disorder: evidence from resting-state functional connectivity. Biological psychiatry, 81(8), 708-717.

Vaghi, M. M., Cardinal, R. N., Apergis-Schoute, A. M., Fineberg, N. A., Sule, A., \& Robbins, T. W. (2019). Action-outcome knowledge dissociates from behavior in obsessive-compulsive disorder following contingency degradation. Biological Psychiatry: Cognitive Neuroscience and Neuroimaging, 4(2), 200-209. 
van’t Riet, J., Sijtsema, S. J., Dagevos, H., \& De Bruijn, G. J. (2011). The importance of habits in eating behaviour. An overview and recommendations for future research. Appetite, 57(3), 585-596.

Verdejo-Garcia, A., Clark, L., Verdejo-Roman, J., Albein-Urios, N., Martinez-Gonzalez, J. M., Gutierrez, B., \& Soriano-Mas, C. (2015). Neural substrates of cognitive flexibility in cocaine and gambling addictions. The British Journal of Psychiatry, 207(2), 158-164.

Verplanken, B., \& Aarts, H. (1999). Habit, attitude, and planned behaviour: Is habit an empty construct or an interesting case of automaticity? European Review of Social Psychology, 10, 101-134.

Verplanken, B., \& Orbell, S. (2003). Reflections on Past Behavior: A Self-Report Index of Habit Strength 1. Journal of applied social psychology, 33(6), 1313-1330.

Wagenmakers, E. J., Love, J., Marsman, M., Jamil, T., Ly, A., Verhagen, J., ... \& Meerhoff, F. (2018). Bayesian inference for psychology. Part II: Example applications with JASP. Psychonomic bulletin \& review, 25(1), 58-76.

Webster, D. M., \& Kruglanski, A. W. (1994). Individual differences in need for cognitive closure. Journal of personality and social psychology, 67(6), 1049.

Whitton, A. E., Henry, J. D., \& Grisham, J. R. (2014). Moral rigidity in obsessive-compulsive disorder: Do abnormalities in inhibitory control, cognitive flexibility and disgust play a role?. Journal of Behavior Therapy and Experimental Psychiatry, 45(1), 152-159.

Wood, W., \& Neal, D. T. (2007). A new look at habits and the habit-goal interface. Psychological review, 114(4), 843.

Zmigrod, L. (2020). The role of cognitive rigidity in political ideologies: theory, evidence, and future directions. Current Opinion in Behavioral Sciences, 34, 34-39. 
Zmigrod, L., Rentfrow, P. J., \& Robbins, T. W. (2018). Cognitive underpinnings of nationalistic ideology in the context of Brexit. Proceedings of the National Academy of Sciences, 115(19), E4532-E4540.

Zmigrod, L., Zmigrod, S., Rentfrow, P. J., \& Robbins, T. W. (2019). The psychological roots of intellectual humility: The role of intelligence and cognitive flexibility. Personality and Individual Differences, 141, 200-208. 


\section{Supplementary Materials}

\section{Combined Analysis: Study $1+2$}

We analysed the combined data from Study 1 and 2 to determine whether the findings from each individual study were reproduced in this larger sample. The aims of this combined analysis were to replicate the relationships between cognitive flexibility, compulsive habitual tendencies and subclinical OCD symptomatology demonstrated in Study 1 and Study 2.

\section{Methods}

\section{Participants}

The data from study 1 and study 2 were combined, resulting in a total of 389 participants for analysis after removal of participants from each sample as outlined above. This combined sample consisted of $54 \%$ males, $45 \%$ females and $1 \%$ other/unspecified, between the ages of 19 and $73(\mathrm{M}=38.090, \mathrm{SD}=11.596)$, all of whom were based in the United States of America. The sample population identified as $69.7 \%$ White, $11.8 \%$ Black or African American, $7.5 \%$ Mixed ethnicity, 5.1\% Asian, 3.3\% Hispanic/Latino, 1.3\% American Indian or Alaska Native, $0.3 \%$ Native American/Pacific Islander, $0.8 \%$ other, $0.3 \%$ unspecified. The highest stages of educational attainment of the sample were as follows: $0.5 \%$ had achieved less than a high school degree, $13.1 \%$ had graduated high school, $21.1 \%$ had completed some school but did not have a degree, $14.1 \%$ had completed a 2-year Associate degree in college, $41.6 \%$ had completed a 4-year Bachelor's degree in college, $8.2 \%$ had a Master's degree, and $1.3 \%$ had a Doctoral or Professional degree. Ethical approval for the study was acquired from the Department of Psychology Ethics Committee of the University of Cambridge. In line with the Declaration of Helsinki (1964), electronic informed consent was obtained from all participants before beginning the survey, and participants were notified that they may terminate their participation in the study at any point. 


\section{Measures}

We administered the HTQ, rated on 7-point Likert scales ranging from "Strongly disagree" to "Strongly agree", along with the additional measures and cognitive tasks, in the form of electronic surveys hosted by Qualtrics Survey Software. As above, these consisted of the revised OCI (Foa et al., 2002), which had a high Cronbach's $\alpha$ value of 0.947, and the AUT (Guilford, 1967; Zmigrod et al., 2019), which had a high Cronbach's $\alpha$ value of 0.818 . The surveys also included two interspersed attention checks, again as in Study 1 and 2.

\section{$\underline{\text { Results }}$}

\section{Correlational analysis}

In order to consider any confounding variables, we examined the correlations between the demographic variables and the psychological variables of interest. Age was found to be negatively correlated with Compulsivity $(r=-0.199, p<0.001)$ and the OCI $(r=-0.260, p<$ $0.001)$, and positively correlated with Aversion to Novelty $(r=0.150, p=0.003)$ and AUT elaboration $(r=0.117, p<0.022)$. Gender differences were present for the 11 -item HTQ, $t(384)$ $=-2.120, p=0.035$; and Aversion to Novelty, $t(384)=-2.321, p=0.021$, with females scoring more highly than males in both. Educational attainment was not significantly correlated with any of the three subscales, AUT measures, or the OCI. Therefore, the demographic variables of age and gender were included as covariates in further analyses.

\section{Relationship between cognitive flexibility and subclinical OCD traits}

In order to evaluate the relationships between cognitive flexibility and subclinical OCD symptomatology, we computed the Pearson's correlations for these variables (see Table S1). As evident in Table S1 and Figure S1, there was a significant negative correlation between AUT Flexibility and the OCI $(r=-0.250, p<0.001)$, as hypothesised, suggesting that 
individuals with increased subclinical OCD traits have a tendency towards increased cognitive rigidity, or decreased cognitive flexibility. The Pearson's $r$ effect size of -0.250 is moderate, as per the individual differences research guidelines set out by Gignac and Szodorai (2016).

To complement the Pearson's correlations, we also examined the Bayes Factors (see Table S1), which demonstrated that the relationship between AUT Flexibility and the OCI possesses an extremely large Bayes Factor of 11392.807 (see Table S1), indicating that the observed data is 11392.807 times more likely under $\mathrm{H}_{1}$ (significant correlation) than $\mathrm{H}_{0}$ (no correlation). As this Bayes Factor value is above 100, it indicates "extreme evidence" for $\mathrm{H}_{1}$, in line with the guidelines from Wagenmakers and colleagues (2018).

\section{Relationship between HTQ and subclinical OCD traits}

There was a significant positive correlation between habitual tendencies, measured by the HTQ, and subclinical OCD traits, measured by the OCI $(r=0.330, p<0.001)$. Of the three HTQ subscales, Compulsivity showed the strongest correlation with the OCI $(r=0.508, p<$ 0.001), and this relationship possessed an extremely large Bayes Factor of $1.639 \times 10^{23}$ (see Table S1), indicating that the observed data is $1.639 \times 10^{23}$ times more likely under $\mathrm{H}_{1}$ (significant correlation) than $\mathrm{H}_{0}$ (no correlation). As this Bayes Factor value is above 100, it indicates "extreme evidence" for $\mathrm{H}_{1}$, in line with the guidelines from Wagenmakers and colleagues (2018).

Table S1. Correlation matrix of habitual tendencies, OCD traits and cognitive measures, including Pearson's correlations and Bayes Factors. 


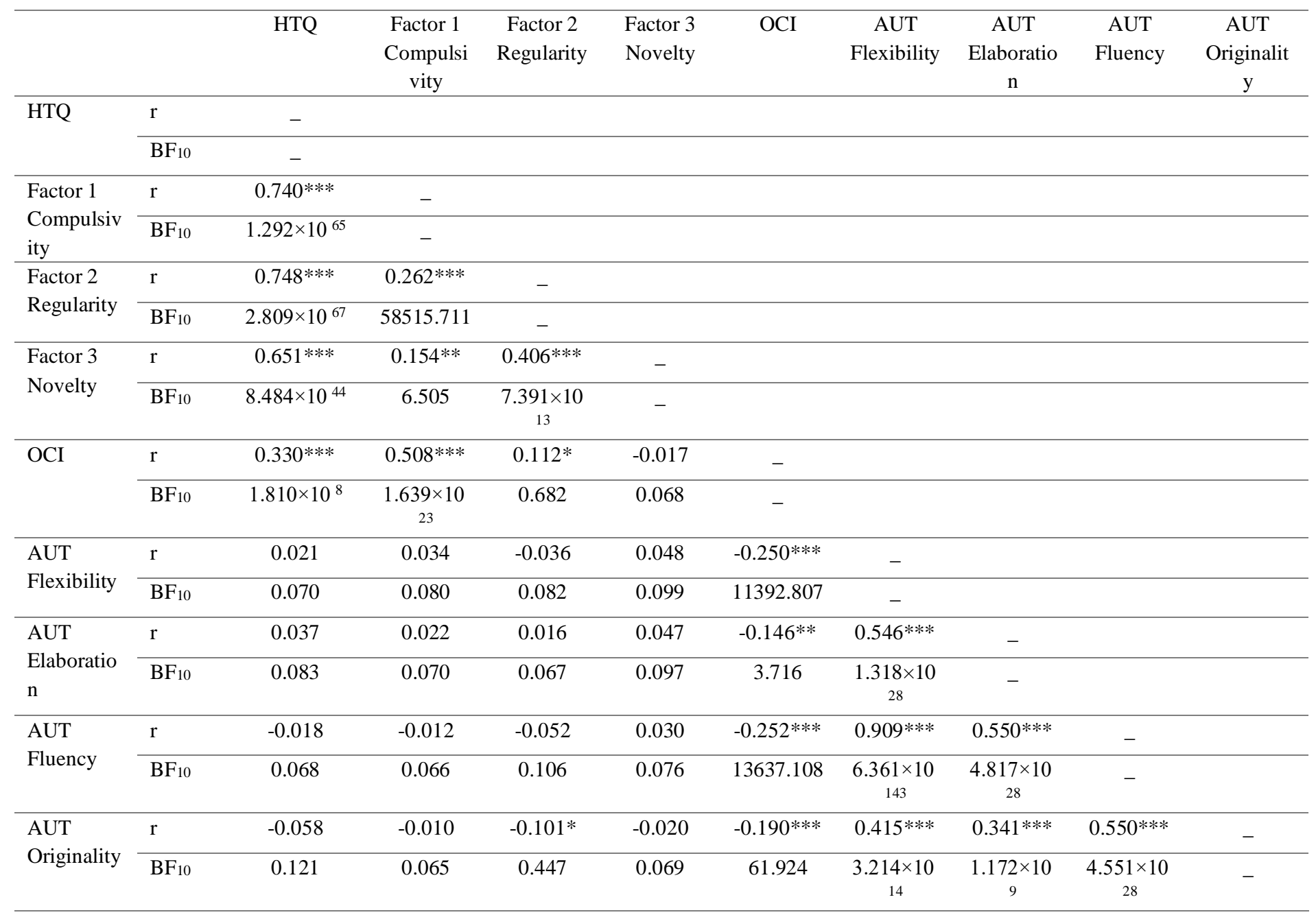

$* \mathrm{p}<0.05, * * \mathrm{p}<0.01, * * * \mathrm{p}<0.001 ; \mathrm{BF}<3=$ Anecdotal evidence; $\mathrm{BF}<10=$ Moderate evidence; $\mathrm{BF}<30=$ Strong evidence $; \mathrm{BF}<100=\mathrm{Very}$ strong evidence; $\mathrm{BF}>100=$ Extremely strong evidence. $\mathrm{HTQ}=$ Habitual Tendencies Questionnaire. AUT $=$ Alternative Uses Task. OCI $=$ Obsessive-Compulsive Inventory. 


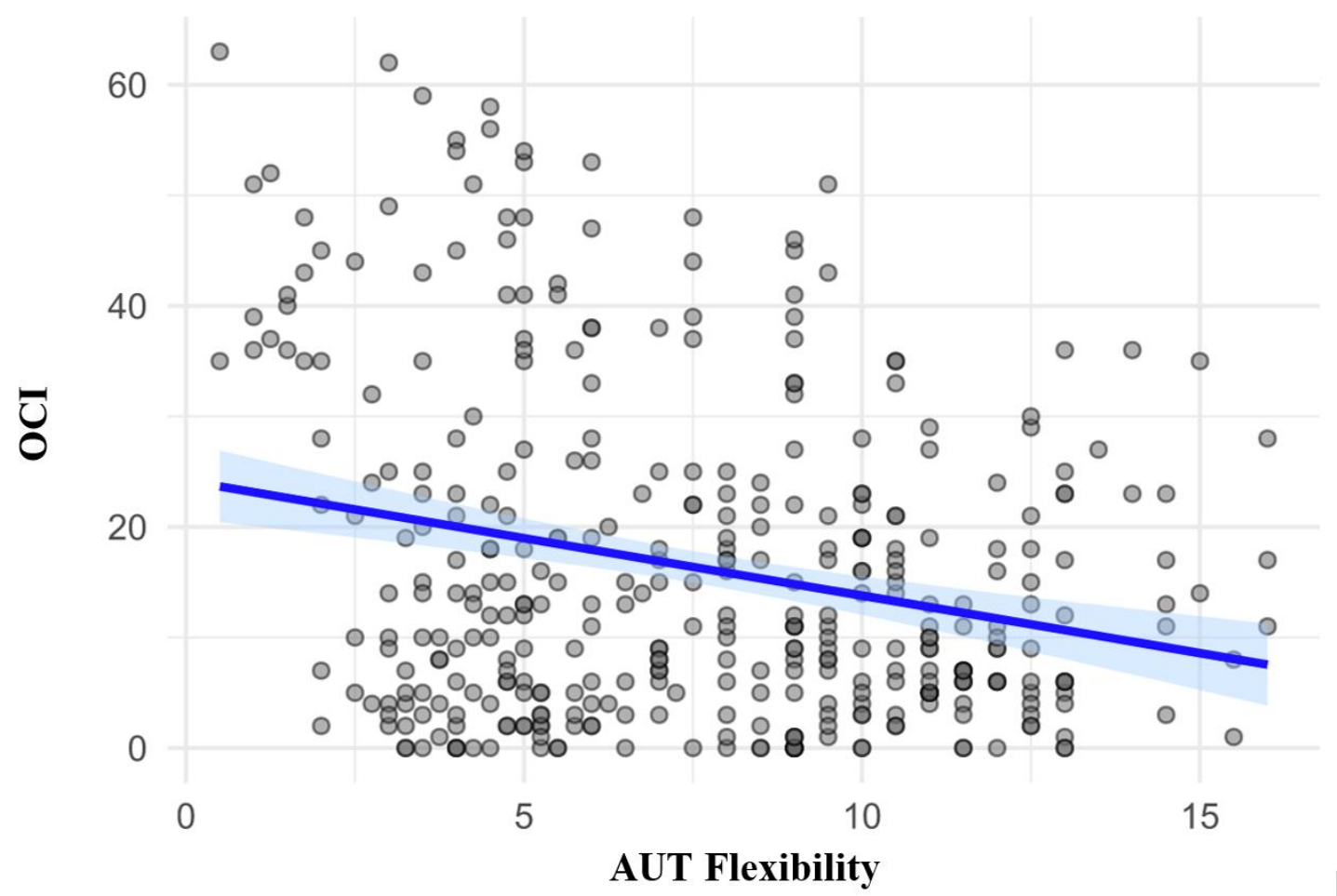

Figure S1. Scatter plot showing correlations and between the Obsessive-Compulsive Inventory (OCI) and the Flexibility component of the Alternative Uses Task (AUT).

\section{Cognitive predictors of subclinical OCD symptomatology}

We then carried out a multiple regression with the three HTQ subscales and the four AUT components as predictors of subclinical OCD symptomatology (see Table S2). Both HTQ Compulsivity and AUT Flexibility emerged as significant predictors of the OCI. Higher Compulsivity and lower cognitive flexibility predicted greater levels of subclinical OCD traits.

As shown in Table S2, HTQ Compulsivity and cognitive flexibility (as measured by the AUT) were significant and unique predictors of the OCI. In order to examine whether there was a significant interaction between these two predictors, hierarchical linear regression was then conducted (see Table S3). In Step 1, the demographic variables age and gender were entered as covariates. As shown in Table S3, age was a significant negative predictor of subclinical OCD symptomatology, such that older participants exhibited lower levels of subclinical OCD symptomatology than younger participants in the present sample. In Step 2, 
HTQ compulsivity and cognitive flexibility (as measured by the AUT) were added, both of which were significant predictors of subclinical OCD symptomatology. HTQ Compulsivity had a positive relationship with the OCI $(\beta=0.485, p<0.001)$ and cognitive flexibility had a negative relationship with the OCI $(\beta=-0.267, p<0.001)$. These independent variables accounted for a significant proportion of the variance in subclinical OCD symptomatology $\left(r^{2}\right.$ $=0.352$ ). In Step 3, the interaction term for HTQ Compulsivity and cognitive flexibility was entered. There was a significant interaction effect between compulsivity and cognitive flexibility, as shown in Table S3, with $\beta=-0.568, p<0.001$. The interaction term increased the $r^{2}$ value to 0.380 , thus accounting for a further $2.8 \%$ of the variance in subclinical OCD symptomatology.

Table S2. Multiple regression with three Habitual Tendencies Questionnaire (HTQ) subscales and four Alternative Uses Task (AUT) components as predictors of subclinical OCD symptomatology (as measured by the Obsessive-Compulsive Inventory, OCI), with demographic variables age and gender as covariates.

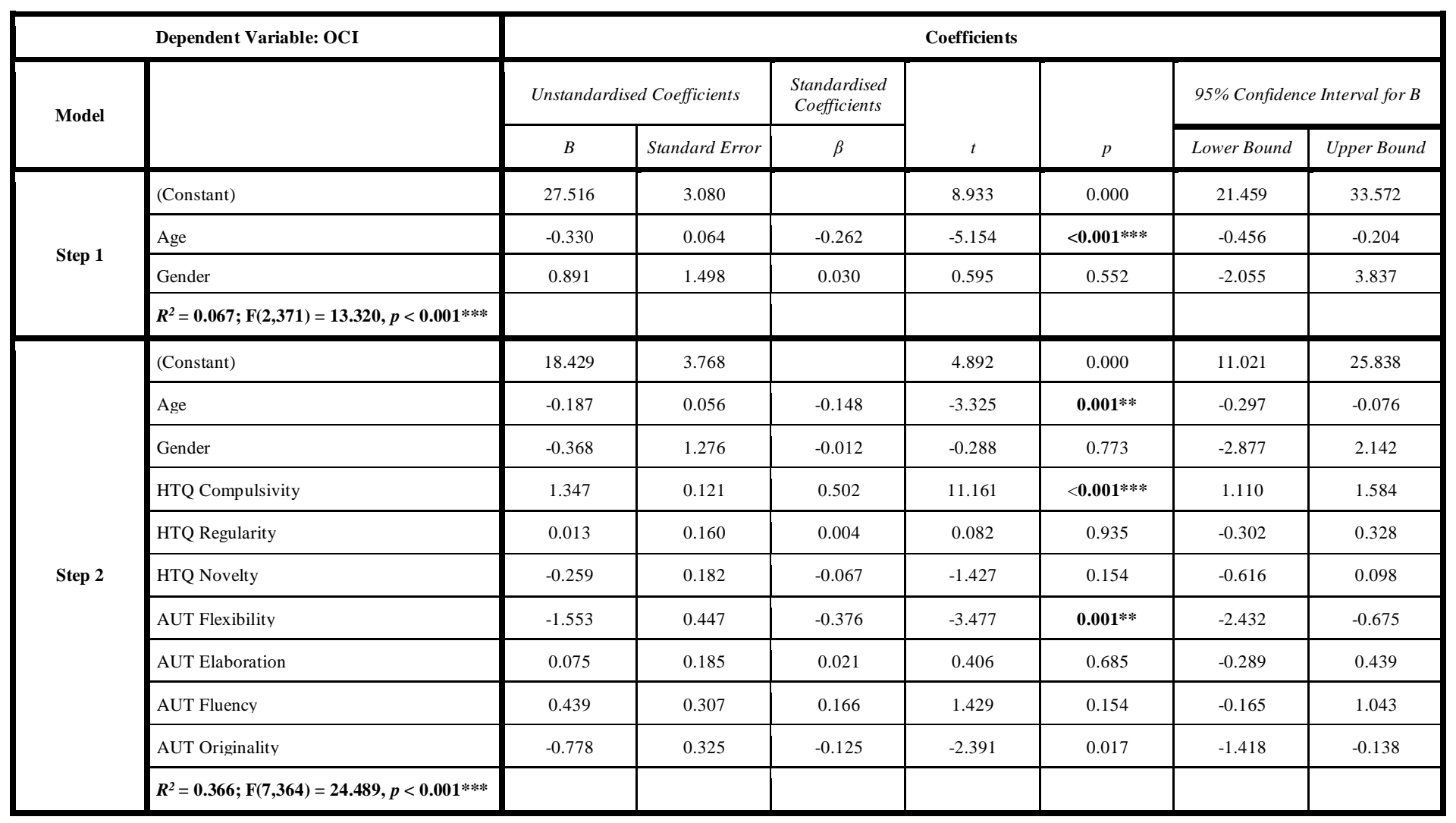


Table S3. 3-step hierarchical linear regression with the Compulsivity subscale of the Habitual Tendencies Questionnaire (HTQ), AUT (Alternative Uses Task) Flexibility and the interaction term between them as predictors of subclinical OCD symptomatology (as measured by the Obsessive-Compulsive Inventory, OCI), with demographic variables age and gender as covariates.

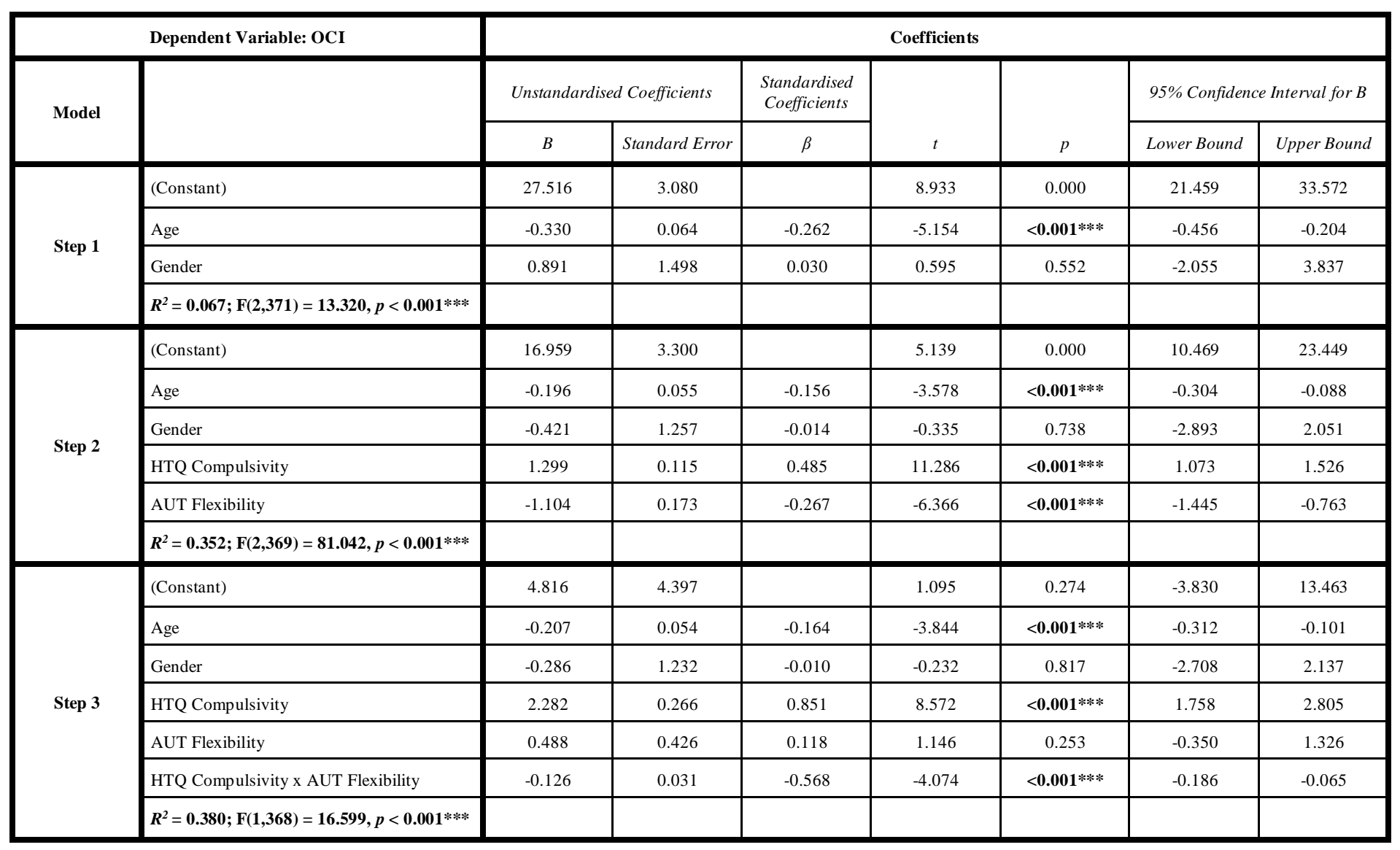

\section{Interaction effects between habitual compulsivity, cognitive flexibility and subclinical}

\section{OCD traits}

We then conducted simple slope analyses (SSA) to investigate the association between cognitive flexibility and subclinical OCD symptomatology at 1 standard deviation (SD) above and below mean HTQ Compulsivity, with age and gender as covariates (see Figure S2a). A significant negative relationship was found between cognitive flexibility and subclinical OCD symptomatology when HTQ Compulsivity was high (at $+1 \mathrm{SD}, b=-1.72, p<0.001$ ), while no significant relationship was found when HTQ Compulsivity was low (at $-1 \mathrm{SD}, b=-0.35, p=$ 
0.17). Carrying out the reciprocal SSA to investigate the association between HTQ Compulsivity and subclinical OCD symptomatology (see Figure S2b) demonstrated that there were significant positive relationships between HTQ Compulsivity and subclinical OCD symptomatology both when cognitive flexibility was high (at $+1 \mathrm{SD}, b=0.88, p<0.001$ ), and when it was low (at $-1 \mathrm{SD}, b=1.77, p<0.001$ ). The interaction effects between HTQ Compulsivity and cognitive flexibility (measured by the AUT) are shown in the filled contour plot in Figure S2c. This shows that the relationship between HTQ Compulsivity and subclinical OCD symptomatology varies depending on cognitive flexibility, such that at high levels but not low levels of HTQ Compulsivity, cognitive flexibility differentiates between high and low levels of subclinical OCD symptomatology. It also shows that the relationship between cognitive flexibility and subclinical OCD symptomatology varies depending on HTQ Compulsivity, such that at both high and low levels of cognitive flexibility, HTQ Compulsivity differentiates between high and low levels of subclinical OCD symptomatology. The highest levels of subclinical OCD traits were observed in participants with high HTQ Compulsivity scores and low AUT Flexibility scores, indicating a compensatory or multiplicative effect, in accordance with the significant interaction effect shown in the hierarchical linear regression (see Table S3). Meanwhile, the lowest levels of subclinical OCD traits were observed in participants with low HTQ Compulsivity scores, regardless of their AUT Flexibility scores. Therefore, high HTQ Compulsivity and low cognitive flexibility are necessary for high levels of subclinical OCD symptomatology, while neither is sufficient independently. These findings are in line with those from the SSA analyses. We used the Johnson-Neyman technique to analyse this interaction further (Johnson and Neyman, 1936), which indicated that the association between cognitive flexibility and OCD was significantly negative at compulsivity scores of 7.75 and above (see Figure S2d). 
a)

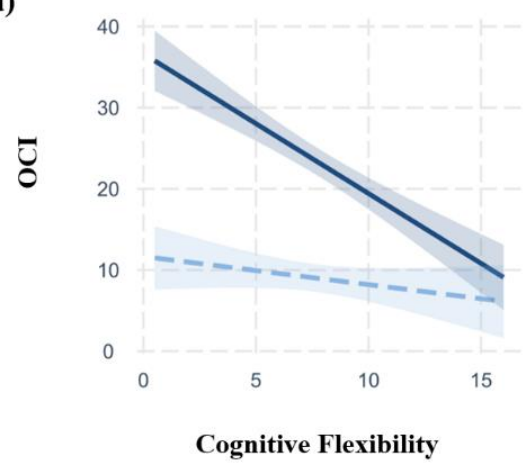

c)

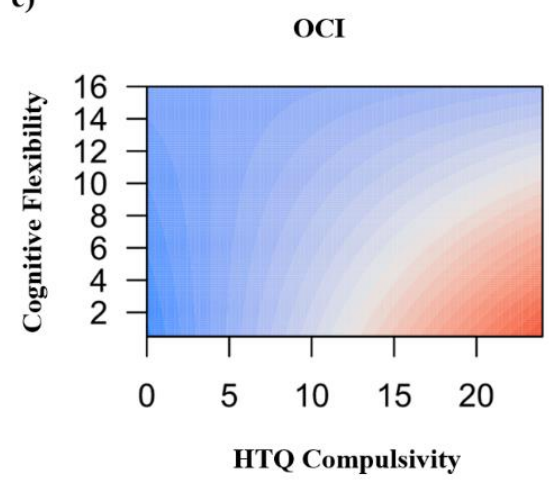

b)

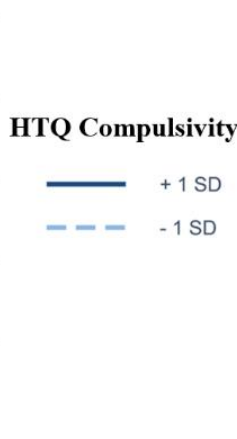

d)

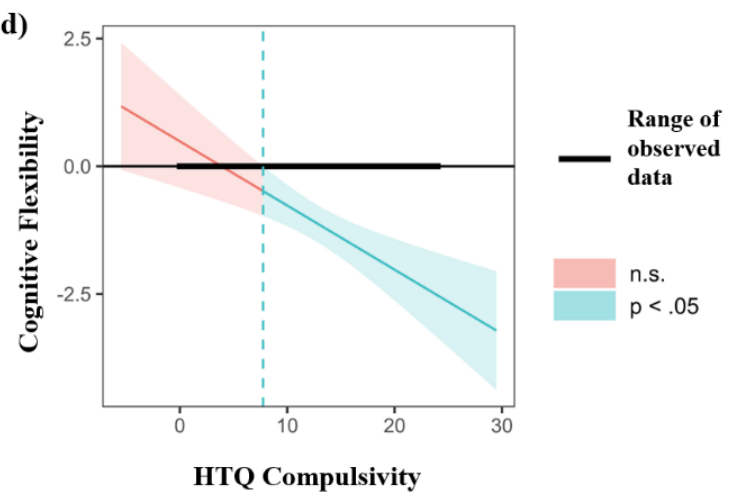

Figure S2. a) Interaction plot between the Compulsivity subscale of the Habitual Tendencies Questionnaire (HTQ), cognitive flexibility (as measured by the Alternative Uses Task) and subclinical OCD symptomatology (as measured by the Obsessive-Compulsive Inventory, OCI) at $1 \mathrm{SD}$ above and below the mean, controlling for age and gender, with cognitive flexibility as the predictor and HTQ Compulsivity as the moderator. b) Interaction plot between the Compulsivity subscale of the Habitual Tendencies Questionnaire (HTQ), cognitive flexibility (as measured by the Alternative Uses Task) and subclinical OCD symptomatology (as measured by the Obsessive-Compulsive Inventory, OCI) at 1 SD above and below the mean, controlling for age and gender, with HTQ Compulsivity as the predictor and cognitive flexibility as the moderator. (Created using the interactions and interplot packages in the statistical software R Studio.) c) Representation of the regression surface predicting subclinical OCD symptomatology (as measured by the Obsessive-Compulsive Inventory, OCI) as a function of the Compulsivity subscale of the Habitual Tendencies Questionnaire (HTQ) and cognitive flexibility (as measured by the Alternative Uses Task), while controlling for age and gender. (Created using the visreg package in the statistical software R Studio.) d) JohnsonNeyman plot showing the conditional relation between cognitive flexibility and OCD symptomatology as a function of the Compulsivity subscale of the Habitual Tendencies Questionnaire (HTQ). The solid diagonal line represents the regression coefficient of cognitive flexibility (as measured by the Alternative Uses Task) for OCD symptomatology along the compulsivity spectrum. The dashed vertical line at a HTQ Compulsivity value of 7.75 represents the transition from significance to non-significance. The width of the regions reflects the $95 \%$ confidence intervals. (Created using the interactions and interplot packages in the statistical software R Studio.) 


\section{Interim Discussion}

In line with our first hypothesis, $\boldsymbol{H} \mathbf{1}$, individuals with lower cognitive flexibility showed increased subclinical OCD symptomatology. In line with our second hypothesis, $\boldsymbol{H} \mathbf{2}$, cognitive flexibility interacted with the Compulsivity subscale of the HTQ to account for $38.0 \%$ of the variance in subclinical OCD symptomatology, demonstrating reproducibility of the findings from Study 1 and 2 in the combined sample. Simple slope analyses (SSA) demonstrated that cognitive inflexibility differentiated between high and low levels of OCD traits when habitual compulsivity was high, but not when it was low, suggesting that habitual compulsivity moderates the negative relationship between cognitive flexibility and subclinical OCD symptomatology. Furthermore, the highest levels of OCD traits were seen in participants with high HTQ Compulsivity scores and low AUT Flexibility scores, suggesting a compensatory effect (see Figure S2). Therefore, both high habitual compulsivity and low cognitive flexibility are necessary for high levels of subclinical OCD symptomatology, while neither is sufficient independently.

In order to make sure that the study was well-powered to detect the present effects, we conducted a conservative post-hoc power analysis $(\alpha=0.001, \mathrm{n}=389)$ using the pwr package in the statistical software R Studio. For the effect sizes, we used the smallest relevant Pearson's correlations (0.250), which reflected the correlations between AUT flexibility and the OCI (see Tables S1). This revealed power of 0.958 , indicating that the sample size was sufficient. Future studies using even larger sample sizes would help to replicate and extend the present findings. 